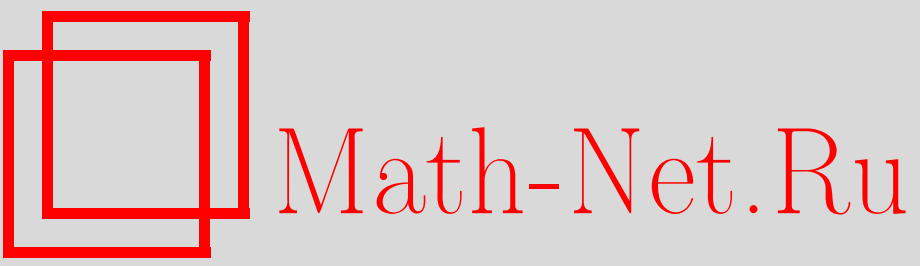

Ю. А. Церковников, Двухвременные температурные функции Грина в кинетической теории и молекулярной гидродинамике. ІІ. Уравнения для систем с парным взаимодействием, ТМФ, 1999, том 119, номер 1, 142-166

DOI: https://doi.org/10.4213/tmf734

Использование Общероссийского математического портала Math-Net.Ru подразумевает, что вы прочитали и согласны с пользовательским соглашением

http: //www.mathnet.ru/rus/agreement

Параметры загрузки:

IP: 54.198 .67 .100

26 апреля 2023 г., $17: 40: 14$ 


\section{ДВУХВРЕМЕННЫЕ ТЕМПЕРАТУРНЫЕ ФУНКЦИИ ГРИНА В КИНЕТИЧЕСКОЙ ТЕОРИИ И МОЛЕКУЛЯРНОЙ ГИДРОДИНАМИКЕ. \\ II. УРАВНЕНИЯ ДЛЯ СИСТЕМ С ПАРНЫМ ВЗАИМОДЕЙСТВИЕМ}

На примере системы частиц с прямым парным взаимодействием рассмотрены два подхода к решению задачи о вычислении корреляционных функций. Первый из них основан на цепочке уравнений, определяющей кинетическое уравнение больцмановского типа. Во втором подходе используется цепочка уравнений молекулярной гидродинамики. Показана эквивалентность обоих подходов в смысле полноты описания рассматриваемой системы. Обсуждаются преимущества подхода, основанного на уравнениях молекулярной гидродинамики.

\section{1. ВВЕДЕНИЕ}

В предыдущей статье [1] была развита общая методика построения цепочек уравнений для неприводимых функций Грина. Вид цепочки уравнений определяется базисными операторами, на которых строятся первые уравнения цепочки. В статье [1] получены также соотношения, позволяющие исходя из неприводимых функций - решений бесконечной цепочки - находить любые корреляционные функции, при этом обеспечивается полное описание исследуемой физической системы.

Данная статья посвяшена дальнейшему развитию предложенной в [1] схемы вычислений на примере квантовой системы с парным взаимодействием. При этом мы убедимся, что в рамках конкретной задачи выбор операторного базиса неоднозначен и от этого выбора зависит эффективность предлагаемого метода. Во втором разделе статьи в качестве базисных выбраны оператор $a_{q}(p)=a_{p-\frac{q}{2}}^{+} a_{p+\frac{q}{2}}$ и сопряженный ему оператор $a_{q}^{+}\left(p^{\prime}\right)=a_{p^{\prime}+\frac{q}{2}}^{+} a_{p^{\prime}-\frac{q}{2}}$. В результате мы получаем цепочку уравнений, определяюшую кинетическое уравнение для функции $\left(\left(a_{q}(p) \mid a_{q}^{+}\left(p^{\prime}\right)\right)\right)_{E}$, пропорциональной фурье-образу вигнеровской функции распределения. Полученные уравнения могут быть использованы для построения уравнений гидродинамики и вычисления кинетических

\footnotetext{
* Математический институт им. В. А. Стеклова РАН, Москва, Россия
} 
коэффициентов. Однако мы не получаем при этом особенных преимуществ по сравнению с обычными методами кинетической теории.

Разработанная в работе [1] методика допускает альтернативный, более эффективньй подход, которому посвяшены третий и последующие разделы данной статьи. В третьем разделе в качестве базиса мы будем использовать набор операторов плотностей сохраняющихся величин (числа частиц $\rho_{q}$, потока частиц $J_{q}^{\alpha}$ и энергии $h_{q}$ ). В результате первым звеном цепочки будут линеаризованные уравнения молекулярной гидродинамики [2], совпадающие по форме с уравнениями обычной гидродинамики, но применимые также и вне гидродинамической области значений градиентов и частот. Последующие уравнения цепочки служат для определения неприводимых относительно операторов $\rho_{q}, J_{q}^{\alpha}$ и $h_{q}$ функций Грина, с помошюю которых выражаются обобшенные кинетические коэффициенты. Как показано в работах [3] и [4], выбор в качестве второго звена уравнений для функций Грина потоков сохраняющихся величин позволяет вычислить кинетические коэффициенты, не прибегая к решению кинетического уравнения.

В работах [4] обобщенные кинетические коэффициенты вычислялись в приближении слабо неидеального газа. Для того чтобы выйти за рамки этого приближения, необходимо включить в рассмотрение уравнения бесконечной цепочки, следующие за уравнениями молекулярной гидродинамики и уравнениями для потоков сохраняющихся величин. Этому посвяшен четвертый раздел статьи. В нем получены уравнения для неприводимых функций Грина, построенных на операторах

$$
a_{q}(p)=a_{p-\frac{q}{2}}^{+} a_{p+\frac{q}{2}}, \quad a_{q}(f)=a_{p_{1}+\frac{k-q}{2}}^{+} a_{p_{2}-\frac{k}{2}}^{+} a_{p_{2}+\frac{k}{2}} a_{p_{1}-\frac{k-q}{2}}, \quad f=\left(p_{1}, p_{2}, k\right),
$$

через которые выражаются обобшенные времена релаксации. То обстоятельство, что с помошью операторов $a_{q}(p)$ и $a_{q}(f)$ выражаются базисные операторы $\rho_{q}, J_{q}^{\alpha}, h_{q}$ и операторы потоков, приводит к необходимости обобщения понятия обратной матрицы, сушествование которой является необходимым условием применимости метода, изложенного в работе [1].

Выбор в качестве первого звена цепочки (в отличие от второго раздела) уравнений молекулярной гидродинамики вовсе не означает, что мы утрачиваем возможность вычисления функций распределения (точнее, их отклонения от равновесного значения), без которых описание исследуемой системы было бы неполным. В пятом разделе показано, что соотношения, полученные в работе [1], позволяют выразить такие функции через “диагональные” неприводимые функции, являющиеся решением бесконечной цепочки уравнений.

В последнем, шестом разделе обсуждаются полученные результаты и возможность дальнейшего развития методики, что позволило бы учесть вклад взаимодействующих мод.

Основными соотношениями работы [1], используемыми в настояшей статье, являются уравнения движения, определяюшие бесконечную последовательность операторов $a(n)(n=1,2, \ldots)$,

$$
\begin{aligned}
i \dot{a}(n) & =[a(n), \mathcal{H}]_{-}=\varepsilon(n, \bar{n}) a(\bar{n})+\nu(n \mid \overline{n+1}) a(\overline{n+1}), \\
-i \dot{a}^{+}\left(n^{\prime}\right) & =\left[\mathcal{H}, a^{+}\left(n^{\prime}\right)\right]_{-}=a^{+}(\bar{n}) \bar{\varepsilon}\left(\bar{n}, n^{\prime}\right)+a^{+}(\overline{n+1}) \bar{\nu}\left(n+1 \mid n^{\prime}\right)
\end{aligned}
$$


и цепочка уравнений для неприводимых функций

$$
\begin{aligned}
& E G_{E}\left(n \mid n^{\prime}\right)=\chi\left(n \mid n^{\prime}\right)+ \\
& \quad+\left\{\left(i \dot{A}(n) \mid A^{+}\left(n^{\prime}\right)\right)+\left(\left(i \dot{A}(n) \mid-i \dot{A}^{+}\left(\bar{n}^{\prime}\right)\right)\right)_{n, E}\right\} \chi^{-1}\left(\bar{n}^{\prime} \mid \bar{n}\right) G_{E}\left(\bar{n} \mid n^{\prime}\right),
\end{aligned}
$$

где при $n=1$ оператор $a(1)$ (или набор операторов, представляемый в виде столбца) является базисным, выбираемым в соответствии с характером рассматриваемой задачи, $A(n)(n=1,2,3, \ldots)$ - последовательность ортогонализованных операторов, получаемая из последовательности $a(n): A(1)=a(1)$,

$$
A(n)=a(n)-\sum_{m=1}^{n-1}\left(a(n) \mid A^{+}\left(\bar{m}^{\prime}\right)\right) \chi^{-1}\left(\bar{m}^{\prime} \mid \bar{m}\right) A(\bar{m}), \quad n=2,3, \ldots
$$

$\chi\left(n \mid n^{\prime}\right)=\left(a(n) \mid a^{+}\left(n^{\prime}\right)\right)_{n-1}=\left(A(n) \mid A^{+}\left(n^{\prime}\right)\right), \quad G_{E}\left(n \mid n^{\prime}\right)=$ $\left(\left(a(n) \mid a^{+}\left(n^{\prime}\right)\right)\right)_{n-1, E}$. Черта над стоящими рядом, не разделенными вертикальной чертой номерами $\bar{n}, \overline{n+1}, \bar{m}, \ldots$ означает свертку по квантовым состояниям и векторным индексам, если $a(n)$ - столбец операторов. Неприводимые функции определяются рекуррентными соотношениями, которые для произвольных операторов $\mathcal{A}$ и $\mathcal{B}^{+}$имеют вид

$$
\begin{aligned}
\left(\left(\mathcal{A} \mid \mathcal{B}^{+}\right)\right)_{n, E}= & \left(\left(\mathcal{A} \mid \mathcal{B}^{+}\right)\right)_{n-1, E}^{-} \\
& -\left(\left(\mathcal{A} \mid A^{+}\left(\bar{n}^{\prime}\right)\right)\right)_{n-1, E} G_{E}^{-1}\left(\bar{n}^{\prime} \mid \bar{n}\right)\left(\left(A(\bar{n}) \mid \mathcal{B}^{+}\right)\right)_{n-1, E}
\end{aligned}
$$

где $n=1,2,3, \ldots,\left(\left(\mathcal{A} \mid \mathcal{B}^{+}\right)\right)_{0, E} \equiv\left(\left(\mathcal{A} \mid \mathcal{B}^{+}\right)\right)_{E}$,

$$
\begin{gathered}
\left(\left(\mathcal{A} \mid \mathcal{B}^{+}\right)\right)_{E}=-i \int_{0}^{\infty} e^{i E t}\left(\mathcal{A}(t) \mid \mathcal{B}^{+}\right) d t \\
\left(\mathcal{A}(t) \mid \mathcal{B}^{+}\right)=i \int_{t}^{\infty}\left\langle\left[\mathcal{A}\left(t^{\prime}\right), \mathcal{B}^{+}\right]_{-}\right\rangle d t^{\prime}
\end{gathered}
$$

$\operatorname{Im} E>0,\left(\mathcal{A}(t) \mid \mathcal{B}^{+}\right)$и $\left(\left(\mathcal{A} \mid \mathcal{B}^{+}\right)\right)_{E}-$ соответственно функция релаксации и ее лапласовский образ. Функции Грина разной степени неприводимости связаны формулой

$$
\begin{aligned}
\left(\left(A(n) \mid \mathcal{B}^{+}\right)\right)_{n-1, E}= & G_{E}\left(n \mid \bar{n}^{\prime}\right) \chi^{-1}\left(\bar{n}^{\prime} \mid \bar{n}\right) \times \\
& \times\left\{\left(A(\bar{n}) \mid \mathcal{B}^{+}\right)+\left(\left(i \dot{A}(\bar{n}) \mid \mathcal{B}^{+}\right)\right)_{n, E}\right\}
\end{aligned}
$$

и ей сопряженной для $\left(\left(\mathcal{A} \mid A^{+}\left(n^{\prime}\right)\right)\right)_{n-1, E}$.

Ссылки на формулы работы [1] будут отмечаться римской цифрой I перед соответствующим номером. Так, приведенным выше формулам соответствуют (по порядку) номера: (I.3.4), (I.3.5) при $b^{+}(n)=a(n),\left(\right.$ I.3.35) при $B^{+}(n)=A^{+}(n)$, (I.3.8), (I.3.23), (I.2.4) и (I.3.29). 


\section{2. КИНЕТИЧЕСКИЕ УРАВНЕНИЯ ДЛЯ ЧАСТИЦ С ПАРНЫМ ВЗАИМОДЕЙСТВИЕМ}

Уравнение движения для одночастичного оператора в гейзенберговском представлении $a_{p}(t)=\exp (i \mathcal{H} t) a_{p} \exp (-i \mathcal{H} t)$ в случае системы бозе- или ферми-частиц с парным взаимодействием имеет вид (в единицах $\hbar=1)$

$$
i \dot{a}_{p}=\left[a_{p}, \mathcal{H}\right]_{-}=\left\{\frac{p^{2}}{2 m}-\mu\right\} a_{p}+\frac{1}{V} \sum_{k, p_{1}} \nu(k) a_{p_{1}-\frac{k}{2}}^{+} a_{p_{1}+\frac{k}{2}} a_{-k+p}
$$

где $a_{p}^{+}$и $a_{p}-$ бозе- или ферми-операторы рождения и уничтожения, $\nu(k)=\nu(|\mathbf{k}|)-$ фурье-образ потенциала взаимодействия между частицами, $k=\mathbf{k}, p=\mathbf{p}$ в случае бозе-системы и $p=(\mathbf{p}, \sigma), \sigma= \pm 1$, для ферми-системы (в этом случае суммирование по $p$ предполагает суммирование как по импульсу $\mathbf{p}$, так и по спиновому индексу $\sigma$ ). В качестве базисного оператора примем $a_{p-\frac{q}{2}}^{+} a_{p+\frac{q}{2}}$. Тогда с учетом уравнения $(2.1)$ из уравнений (1.1) получаем цепочку уравнений движения для операторов

$$
\begin{aligned}
a(1) & \equiv a_{q}(p)=a_{p-\frac{q}{2}}^{+} a_{p+\frac{q}{2}} \\
a(2) & \equiv a_{q}(f)=a_{q}\left(p_{1}, p_{2}, k\right)=a_{p_{1}+\frac{k-q}{2}}^{+} a_{p_{2}-\frac{k}{2}}^{+} a_{p_{2}+\frac{k}{2}} a_{p_{1}-\frac{k-q}{2}}, \\
a(3) & \equiv a_{q}(g)=a_{q}\left(p_{1}, p_{2}, p_{3} ; k, k_{1}\right)= \\
& =a_{p_{1}+\frac{k+k_{1}-q}{2}}^{+} a_{p_{2}-\frac{k}{2}}^{+} a_{p_{3}-\frac{k_{1}}{2}}^{+} a_{p_{3}+\frac{k_{1}}{2}} a_{p_{2}+\frac{k}{2}} a_{p_{1}-\frac{k+k_{1}-q}{2}},
\end{aligned}
$$

где $q \equiv \mathbf{q}, k \equiv \mathbf{k}, k_{1} \equiv \mathbf{k}_{1}, p_{i}=\left(\mathbf{p}_{i}, \sigma_{i}\right)$ в случае ферми-системы и $f, g, \ldots-$ сокращенные обозначения для соответствуюших совокупностей индексов $p$ и $k$. В первых двух уравнениях цепочки (1.1) (при $n=1,2)$ коэффициенты имеют вид

$$
\begin{aligned}
\varepsilon\left(1,1^{\prime}\right) & \equiv \varepsilon_{q}\left(p, p^{\prime}\right)=\varepsilon_{q}(p) \delta\left(p, p^{\prime}\right), \quad \varepsilon_{q}(p)=\frac{\left(p+\frac{q}{2}\right)^{2}-\left(p-\frac{q}{2}\right)^{2}}{2 m}=\frac{(p, q)}{m} \\
\nu(1 \mid 2) & \equiv \nu(p \mid f)=\nu\left(p \mid p_{1}, p_{2}, k\right)=\frac{\nu(k)}{V}\left(\delta_{p, p_{1}+\frac{k}{2}}-\delta_{p, p_{1}-\frac{k}{2}}\right) \\
\varepsilon\left(2 \mid 2^{\prime}\right) & \equiv \varepsilon_{q}\left(f, f^{\prime}\right)=\varepsilon_{q}(f) \delta_{q}\left(f, f^{\prime}\right)+\nu\left(f, f^{\prime}\right) \\
\varepsilon_{q}(f) & =\frac{\left(p_{1},-k+q\right)+\left(p_{2}, k\right)}{m} \\
\nu\left(f, f^{\prime}\right) & \equiv \nu\left(p_{1}, p_{2}, k ; p_{1}^{\prime}, p_{2}^{\prime}, k^{\prime}\right)=\nu\left(p_{1} \mid p_{1}^{\prime}, p_{2}^{\prime}, k^{\prime}-k\right) \delta_{p_{1}+p_{2}, p_{1}^{\prime}+p_{2}^{\prime}}
\end{aligned}
$$

И

$$
\begin{aligned}
\nu\left(2 \mid 3^{\prime}\right) & \equiv \nu\left(f \mid g^{\prime}\right)=\nu\left(p_{1}, p_{2}, k \mid p_{1}^{\prime}, p_{2}^{\prime}, p_{3}^{\prime} ; k^{\prime} k_{1}^{\prime}\right)= \\
& =\nu\left(p_{1} \mid p_{1}^{\prime}, p_{2}^{\prime}, k_{1}^{\prime}\right) \delta_{p_{2}, p_{2}^{\prime}} \delta_{k, k^{\prime}}+\nu\left(p_{2} \mid p_{1}^{\prime}, p_{2}^{\prime}, k_{1}^{\prime}\right) \delta_{p_{1}, p_{2}^{\prime}} \delta_{-k+q, k^{\prime}}
\end{aligned}
$$

6 Теоретическая и математическая физика, т. 119, № 1, 1999 г. 
В соотношениях $(2.3)-(2.6)$ функции $\delta\left(p, p^{\prime}\right)$ и $\delta_{q}\left(f, f^{\prime}\right)$ имеют вид

$$
\begin{aligned}
\delta\left(1,1^{\prime}\right) \equiv & \delta\left(p, p^{\prime}\right)=\delta_{p, p^{\prime}} \\
\delta\left(2,2^{\prime}\right) \equiv & \delta_{q}\left(f, f^{\prime}\right)=\delta_{q ; f, f^{\prime}}=\frac{1}{2\left(1+\delta_{\sigma_{1} \sigma_{2}}\right)} \delta_{p_{1}+p_{2}, p_{1}^{\prime}+p_{2}^{\prime}} \times \\
& \times\left\{\delta_{p_{1}, p_{1}^{\prime}} \delta_{k, k^{\prime}}+\delta_{p_{2} p_{1}^{\prime}} \delta_{-k+q, k^{\prime}} \pm\right. \\
& \pm \delta_{p_{1}-p_{2}+\frac{q}{2}, k^{\prime}} \delta_{k, p_{1}^{\prime}-p_{2}^{\prime}+\frac{q}{2}} \pm \delta_{p_{2}-p_{1}+\frac{q}{2}, k^{\prime}} \delta_{\left.k, p_{2}^{\prime}-p_{1}^{\prime}+\frac{q}{2}\right\}}
\end{aligned}
$$

Знак минус в правой части (2.7) соответствует ферми-операторам. В случае бозе-системы в знаменателе следует заменить $\delta_{\sigma_{1}, \sigma_{2}}$ на 1 . Символы Кронекера, связываюшие в формулах $(2.6),(2.7)$ индексы $p$, связывают и индексы $\mathbf{p}$ и $\sigma$. Так, $\delta_{k, k^{\prime}}=\delta_{\mathbf{k}, \mathbf{k}^{\prime}}$, но

$$
\delta_{p, p^{\prime}}=\delta_{\mathbf{p}, \mathbf{p}^{\prime}} \delta_{\sigma, \sigma^{\prime}}, \quad \delta_{p_{1}-p_{2}+\frac{q}{2}, k^{\prime}}=\delta_{\mathbf{p}_{1}-\mathbf{p}_{2}+\frac{\mathbf{q}}{2}, \mathbf{k}^{\prime}} \delta_{\sigma_{1}, \sigma_{2}}
$$

и т.д., $\nu(k)=\nu(|\mathbf{k}|), \quad \nu\left(p_{1}-p_{2}\right)=\nu\left(\left|\mathbf{p}_{1}-\mathbf{p}_{2}\right|\right) \delta_{\sigma_{1} \sigma_{2}}$ и т.д. Обобщенные $\delta$-символы $\delta\left(p, p^{\prime}\right), \delta\left(f, f^{\prime}\right), \ldots$ обладают симметрией, свойственной операторам $a_{q}(p), a_{q}(f), \ldots$ (как, например, $a_{q}(f)$ при замене $p_{1} \rightleftarrows p_{2}, k \rightarrow-k+q$ ), и всеми необходимыми свойствами, отраженными в формулах (I.3.12)-(I.3.14).

Ортогонализуя в соответствии с (1.4), (І.3.8) последовательность операторов (2.2):

$$
\begin{aligned}
A(1) & \equiv A_{q}(p)=a_{q}(p), \quad A^{+}\left(1^{\prime}\right) \equiv A_{q}^{+}\left(p^{\prime}\right)=a_{q}^{+}\left(p^{\prime}\right), \\
A(2) & \equiv A_{q}(f)=a_{q}(f)-\left(a_{q}(f) \mid a_{q}^{+}\left(\bar{p}^{\prime}\right)\right) \chi_{q}^{-1}\left(\bar{p}^{\prime} \mid \bar{p}\right) a_{q}(\bar{p}), \\
A^{+}\left(2^{\prime}\right) & \equiv A_{q}^{+}\left(f^{\prime}\right)=a_{q}^{+}\left(f^{\prime}\right)-a_{q}^{+}\left(\bar{p}^{\prime}\right) \chi_{q}^{-1}\left(\bar{p}^{\prime} \mid \bar{p}\right)\left(a_{q}(\bar{p}) \mid a_{q}^{+}\left(f^{\prime}\right)\right),
\end{aligned}
$$

где

$$
\begin{aligned}
\chi_{q}\left(p \mid p^{\prime}\right) & =\left(a_{q}(p) \mid a_{q}^{+}\left(p^{\prime}\right)\right), \\
\chi_{q}\left(f \mid f^{\prime}\right) & =\left(A_{q}(f) \mid A_{q}^{+}\left(f^{\prime}\right)\right)= \\
& =\left(a_{q}(f) \mid a_{q}^{+}\left(f^{\prime}\right)\right)-\left(a_{q}(f) \mid a_{q}^{+}\left(\bar{p}^{\prime}\right)\right) \chi_{q}^{-1}\left(\bar{p}^{\prime} \mid \bar{p}\right)\left(a_{q}(\bar{p}) \mid a_{q}^{+}\left(f^{\prime}\right)\right),
\end{aligned}
$$

для неприводимых (диагональных по номерам $n=1,2, \ldots$ ) функций

$$
\begin{aligned}
G_{q, E}\left(1 \mid 1^{\prime}\right) & \equiv G_{q, E}\left(p \mid p^{\prime}\right)=\left(\left(a_{q}(p) \mid a_{q}^{+}\left(p^{\prime}\right)\right)\right)_{E} \\
G_{E}\left(2 \mid 2^{\prime}\right) & \equiv G_{q, E}\left(f \mid f^{\prime}\right)=\left(\left(A_{q}(f) \mid A_{q}^{+}\left(f^{\prime}\right)\right)\right)_{1, E}=\left(\left(a_{q}(f) \mid a_{q}^{+}\left(f^{\prime}\right)\right)\right)_{1, E}, \ldots
\end{aligned}
$$

будем иметь согласно соотношениям (1.3), (I.3.16), (I.3.18) уравнения

$$
\begin{aligned}
E G_{q, E}\left(p \mid p^{\prime}\right)= & \chi_{q}\left(p \mid p^{\prime}\right)+\omega_{q}(p, \bar{p}) G_{q, E}\left(\bar{p} \mid p^{\prime}\right)+ \\
& +\nu(p \mid \bar{f}) G_{q, E}\left(\bar{f} \mid \bar{f}^{\prime}\right) \bar{\nu}\left(\bar{f}^{\prime} \mid \bar{p}^{\prime}\right) \chi_{q}^{-1}\left(\bar{p}^{\prime} \mid \bar{p}\right) G_{q, E}\left(\bar{p} \mid p^{\prime}\right), \\
E G_{q, E}\left(f \mid f^{\prime}\right)= & \chi_{q}\left(f \mid f^{\prime}\right)+\omega_{q}(f, \bar{f}) G_{q, E}\left(\bar{f} \mid f^{\prime}\right)+ \\
& +\nu(f \mid \bar{g}) G_{q, E}\left(\bar{g} \mid \bar{g}^{\prime}\right) \bar{\nu}\left(\bar{g}^{\prime} \mid \bar{f}^{\prime}\right) \chi_{q}^{-1}\left(\bar{f}^{\prime} \mid \bar{f}\right) G_{q, E}\left(\bar{f} \mid f^{\prime}\right) .
\end{aligned}
$$


Уравнения для функций $G_{q, E}\left(g \mid g^{\prime}\right), \ldots$ строятся по такой же схеме. Для последовательности операторов (2.2) одновременно существуют как функции $\chi^{-1}\left(n \mid n^{\prime}\right)$, так и функции $\eta^{-1}\left[n \mid n^{\prime}\right] \quad\left(\eta\left[n \mid n^{\prime}\right]=\left\langle\left[A[n], A^{+}\left[n^{\prime}\right]\right]_{-}\right\rangle\right.$, см. уравнения (I.4.1)-(I.4.3)). В этом случае мы можем, воспользовавшись формулами (I.4.20), (I.4.9), исключить из функций $\omega\left(n, n^{\prime}\right)(\mathrm{I} .3 .18)$ неизвестные скалярные произведения $\left(\mathcal{A} \mid \mathcal{B}^{+}\right)(1.6)$, заменив их усредненными коммутаторами $\left\langle\left[\mathcal{A}, \mathcal{B}^{+}\right]_{-}\right\rangle$. В результате будем иметь

$$
\begin{aligned}
\omega_{q}\left(p, p^{\prime}\right)= & \omega_{q}\left[p, p^{\prime}\right]-\nu(p \mid \bar{f}) \chi_{q}\left(\bar{f} \mid \bar{f}^{\prime}\right) \bar{\nu}\left(\bar{f}^{\prime} \mid \bar{p}^{\prime}\right) \eta_{q}^{-1}\left[\bar{p}^{\prime} \mid p^{\prime}\right], \\
\omega_{q}\left(f, f^{\prime}\right)= & \omega_{q}\left[f, f^{\prime}\right]-\nu(f \mid \bar{g}) \chi_{q}\left(\bar{g} \mid \bar{g}^{\prime}\right) \bar{\nu}\left(\bar{g}^{\prime} \mid \bar{f}^{\prime}\right) \eta_{q}^{-1}\left[\bar{f}^{\prime} \mid f^{\prime}\right]+ \\
& +\chi_{q}\left(f \mid \bar{f}^{\prime}\right) \bar{\nu}\left(\bar{f}^{\prime} \mid \bar{p}^{\prime}\right) \eta_{q}^{-1}\left[\bar{p}^{\prime} \mid \bar{p}\right] \nu\left(\bar{p} \mid f^{\prime}\right), \quad \ldots,
\end{aligned}
$$

где

$$
\begin{aligned}
\omega_{q}\left[p, p^{\prime}\right]= & \varepsilon_{q}\left(p, p^{\prime}\right)+\nu(p \mid \bar{f})\left\langle\left[a_{q}(\bar{f}), a_{q}^{+}\left(\bar{p}^{\prime}\right)\right]\right\rangle \eta_{q}^{-1}\left[\bar{p}^{\prime} \mid p^{\prime}\right], \\
\omega_{q}\left[f, f^{\prime}\right]= & \varepsilon_{q}\left(f, f^{\prime}\right)+\nu(f \mid \bar{g})\left\langle\left[a_{q}(\bar{g}), A_{q}^{+}\left[\bar{f}^{\prime}\right]\right]\right\rangle \eta_{q}^{-1}\left[\bar{f}^{\prime} \mid f^{\prime}\right]- \\
& -\left\langle\left[a_{q}(f), a_{q}^{+}\left(\bar{p}^{\prime}\right)\right]\right\rangle \eta_{q}^{-1}\left[\bar{p}^{\prime} \mid \bar{p}\right] \nu\left(\bar{p} \mid f^{\prime}\right), \quad \ldots
\end{aligned}
$$

Восприимчивости $\chi_{q}\left(p \mid p^{\prime}\right), \chi_{q}\left(f \mid f^{\prime}\right), \ldots$ удовлетворяют согласно (І.4.16) цепочке уравнений

$$
\begin{aligned}
& \omega_{q}[p, \bar{p}] \chi_{q}\left(\bar{p} \mid p^{\prime}\right)- \\
& \quad-\nu(p \mid \bar{f}) \chi_{q}\left(\bar{f} \mid \bar{f}^{\prime}\right) \bar{\nu}\left(\bar{f}^{\prime} \mid \bar{p}^{\prime}\right) \eta_{q}^{-1}\left[\bar{p}^{\prime} \mid \bar{p}\right] \chi_{q}\left(\bar{p} \mid p^{\prime}\right)=\eta_{q}\left[p \mid p^{\prime}\right], \\
& \omega_{q}[f, \bar{f}] \chi_{q}\left(\bar{f} \mid f^{\prime}\right)- \\
& \quad-\nu(f \mid \bar{g}) \chi_{q}\left(\bar{g} \mid \bar{g}^{\prime}\right) \bar{\nu}\left(\bar{g}^{\prime} \mid \bar{f}^{\prime}\right) \eta_{q}^{-1}\left[\bar{f}^{\prime} \mid \bar{f}\right] \chi_{q}\left(\bar{f} \mid f^{\prime}\right)=\eta_{q}\left[f \mid f^{\prime}\right],
\end{aligned}
$$

где

$$
\begin{aligned}
\eta_{q}\left[p \mid p^{\prime}\right] & =\left\langle\left[a_{q}(p), a_{q}^{+}\left(p^{\prime}\right)\right]_{-}\right\rangle=\left(n_{p-\frac{q}{2}}-n_{p+\frac{q}{2}}\right) \delta_{p, p^{\prime}}=n_{q}(p) \delta_{p, p^{\prime}} \\
\eta_{q}\left[f \mid f^{\prime}\right] & =\left\langle\left[a_{q}(f), a_{q}^{+}\left(f^{\prime}\right)\right]\right\rangle_{1}=\left\langle\left[A_{q}[f], A_{q}^{+}\left[f^{\prime}\right]\right]_{-}\right\rangle= \\
& =\left\langle\left[A_{q}[f], a_{q}^{+}\left(f^{\prime}\right)\right]_{-}\right\rangle \approx n_{q}(f) 2\left(1+\delta_{\sigma_{1}, \sigma_{2}}\right) \delta_{q}\left(f, f^{\prime}\right) .
\end{aligned}
$$

По аналогии с формулами (2.8) имеем

$$
A_{q}[p]=a_{q}(p), \quad A_{q}[f]=a_{q}(f)-\left\langle\left[a_{q}(f), a_{q}^{+}\left(\bar{p}^{\prime}\right)\right]_{-}\right\rangle \eta_{q}^{-1}\left[\bar{p}^{\prime} \mid \bar{p}\right] a_{q}(\bar{p}) .
$$

Очевидно, что $\eta_{q}\left[p \mid p^{\prime}\right]=\eta_{q}\left(p \mid p^{\prime}\right)$, но $\eta_{q}\left[f \mid f^{\prime}\right] \neq \eta_{q}\left(f \mid f^{\prime}\right)=\left\langle\left[A_{q}(f), A_{q}^{+}\left(f^{\prime}\right)\right]_{-}\right\rangle$. Последнее приближенное равенство в $\left(2.17^{\prime}\right)$ соответствует нулевому приближению по взаимодействию. Функция $\delta_{q}\left(f, f^{\prime}\right)$ определяется соотношением $(2.7)$, а

$$
\begin{aligned}
n_{q}(f) \equiv & n_{q}\left(p_{1}, p_{2}, k\right)=n_{p_{1}+\frac{k-q}{2}} n_{p_{2}-\frac{k}{2}}\left(1 \pm n_{p_{2}+\frac{k}{2}}\right)\left(1 \pm n_{p_{1}-\frac{k-q}{2}}\right)- \\
& -\left(1 \pm n_{p_{1}+\frac{k-q}{2}}\right)\left(1 \pm n_{p_{2}-\frac{k}{2}}\right) n_{p_{2}+\frac{k}{2}} n_{p_{1}-\frac{k-q}{2}}
\end{aligned}
$$


и $n_{p}=\left\langle a_{p}^{+} a_{p}\right\rangle$. Чтобы вычислить средние вида $\left\langle a_{p_{1}}^{+} \ldots a_{p_{n}}^{+} a_{p_{n}^{\prime}} \ldots a_{p_{1}^{\prime}}\right\rangle$, входящие в правые части выражений для коэффициентов (2.12)-(2.15), следует воспользоваться цепочкой уравнений для обычных коммутаторных (антикоммутаторных) функций Грина (I.2.1), построенных по предлагаемой схеме на основе последовательности операторов $a_{p}, a_{p_{1}-\frac{k}{2}}^{+} a_{p_{1}+\frac{k}{2}} a_{-k+p}, \ldots$, где $a(1)=a_{p}$ - одночастичный базисный оператор, удовлетворяющий уравнению движения (2.1).

Уравнения (2.10), (2.11) и (2.16), (2.17) представляют собой первые два уравнения бесконечной цепочки соответственно для релаксационных функций Грина (1.5) и восприимчивостей $\chi\left(n \mid n^{\prime}\right)$. Они являются основой построения кинетического уравнения для релаксационной функции Грина $G_{q, E}\left(p \mid p^{\prime}\right)=\left(\left(a_{q}(p) \mid a_{q}^{+}\left(p^{\prime}\right)\right)\right)_{E}$, которая в силу уравнения (I.2.12) связана с вариацией фурье-образа функции Вигнера

$$
f_{t}(p, x)=\frac{1}{N} \sum_{q} \exp i(\mathbf{q}, \mathbf{x})\left\langle a_{p-\frac{q}{2}}^{+} a_{p+\frac{q}{2}}\right\rangle_{t} .
$$

Если найдены диагональные по номеру $n$ функции $G_{q, E}\left(p \mid p^{\prime}\right), G_{q, E}\left(f \mid f^{\prime}\right), \ldots$ и $\chi_{q}\left(p \mid p^{\prime}\right), \chi_{q}\left(f \mid f^{\prime}\right), \ldots$, все другие необходимые функции можно вычислить с помошью соотношений (1.7) или (I.3.33), (І.3.34) и определения неприводимых функций (1.5), например,

$$
\begin{aligned}
& \left(\left(A_{q}(f) \mid a_{q}^{+}\left(p^{\prime}\right)\right)\right)_{E}=G_{q, E}\left(f \mid \bar{f}^{\prime}\right) \bar{\nu}\left(\bar{f}^{\prime} \mid \bar{p}^{\prime}\right) \chi_{q}^{-1}\left(\bar{p}^{\prime} \mid \bar{p}\right) G_{q, E}\left(\bar{p} \mid p^{\prime}\right) \\
& \left(\left(a_{q}(p) \mid A_{q}^{+}\left(f^{\prime}\right)\right)\right)_{E}=G_{q, E}\left(p \mid \bar{p}^{\prime}\right) \chi_{q}^{-1}\left(\bar{p}^{\prime} \mid \bar{p}\right) \nu(\bar{p} \mid \bar{f}) G_{q, E}\left(\bar{f} \mid f^{\prime}\right)
\end{aligned}
$$

и

$$
\begin{aligned}
\left(\left(A_{q}(f) \mid A_{q}^{+}\left(f^{\prime}\right)\right)\right)_{E}= & G_{q, E}\left(f \mid f^{\prime}\right)+G_{q, E}\left(f \mid \bar{f}^{\prime}\right) \bar{\nu}\left(\bar{f}^{\prime} \mid \bar{p}^{\prime}\right) \chi_{q}^{-1}\left(\bar{p}^{\prime} \mid \bar{p}\right) \times \\
& \times G_{q, E}\left(\bar{p} \mid \bar{p}^{\prime}\right) \chi_{q}^{-1}\left(\bar{p}^{\prime} \mid \bar{p}\right) \nu(\bar{p} \mid \bar{f}) G_{q, E}\left(\bar{f} \mid f^{\prime}\right)
\end{aligned}
$$

(см. также формулы (І.3.39)-(І.3.41)). Поскольку плотности сохраняющихся величин выражаются через операторы $a_{q}(p)$ и $a_{q}(f)$ :

$$
\begin{aligned}
\rho_{q} & =\frac{1}{\sqrt{N}} \sum_{p} a_{q}(p), \quad J_{q}^{\alpha}=\frac{1}{\sqrt{N}} \sum_{p} \frac{p_{\alpha}}{m} a_{q}(p), \quad h_{q}=h_{q}^{\mathrm{kin}}+h_{q}^{\mathrm{int}}, \\
h_{q}^{\mathrm{kin}} & =\frac{1}{\sqrt{N}} \sum_{p}\left(\frac{p^{2}}{2 m}-\frac{q^{2}}{8 m}\right) a_{q}(p), \quad h_{q}^{\mathrm{int}}=\frac{1}{2 \sqrt{N} V} \sum_{f=\left(p_{1}, p_{2}, k\right)} \nu(k) a_{q}(f),
\end{aligned}
$$

то с помощью соотношений (2.19), (2.20) можно получить выражения для функций Грина $\left(\left(\rho_{q} \mid \rho_{-q}\right)\right)_{E},\left(\left(h_{q} \mid h_{-q}\right)\right)_{E},\left(\left(J_{q}^{\alpha} \mid J_{-q}^{\beta}\right)\right)_{E}, \quad\left(\left(h_{q} \mid \rho_{-q}\right)\right)_{E}$ и т.д. через неприводимые функции $G_{q, E}\left(p \mid p^{\prime}\right)$ и $G_{q, E}\left(f \mid f^{\prime}\right)$.

Полученные уравнения применимы при любых взаимодействиях и плотностях. В случае слабо неидеального газа из них легко получаются уже известные результаты. Так, заменяя в уравнении (2.10) функции $G_{q, E}\left(f \mid f^{\prime}\right)$ и $\chi_{q}\left(p \mid p^{\prime}\right)$ их значениями для идеального газа $G_{q, E}^{\mathrm{id}}\left(f \mid f^{\prime}\right)$ и $\chi_{q}^{\mathrm{id}}\left(p \mid p^{\prime}\right)$ и полагая $E=i \varepsilon$, получим для $G_{q, E}\left(p \mid p^{\prime}\right)$ 
линеаризованное кинетическое уравнение. Если взаимодействие нельзя считать малым (например, когда газ малой плотности состоит из молекул типа “твердых шаров" или потенциал сингулярен), то в уравнении (2.10) следует положить $G_{q, E}\left(f \mid f^{\prime}\right) \approx$ $G_{q, E}^{(0)}\left(f \mid f^{\prime}\right)$, где $G_{q, E}^{(0)}\left(f \mid f^{\prime}\right)$ - решение уравнения, получаемого из уравнения (2.11), если пренебречь членами второго порядка по взаимодействию, входящими в (2.11) явно и через $\omega_{q}\left(f \mid f^{\prime}\right)(2.13),(2.15)$, а в операторе $a_{q}(g)(2.2)$ в правой части $(2.15)$ произвести все допустимые спаривания. В результате для функции $G_{q, E}^{(0)}\left(f \mid f^{\prime}\right)$ и (в том же приближении) для функции $\chi_{q}\left(f \mid f^{\prime}\right) \approx \chi_{q}^{(0)}\left(f \mid f^{\prime}\right)$ (см. (2.17) будем иметь уравнения

$$
\begin{gathered}
\left(E-\omega_{q}(f)\right) G_{q, E}^{(0)}\left(f \mid f^{\prime}\right)=\chi_{q}^{(0)}\left(f \mid f^{\prime}\right)+\mathcal{V}_{q}(f \mid \bar{f}) G_{q, E}^{(0)}\left(\bar{f} \mid f^{\prime}\right) \\
\chi_{q}^{(0)}\left(f \mid f^{\prime}\right) \omega_{q}\left(f^{\prime}\right)+\chi_{q}^{(0)}(f \mid \bar{f}) \overline{\mathcal{V}}_{q}\left(\bar{f} \mid f^{\prime}\right)=\eta_{q}\left[f \mid f^{\prime}\right]
\end{gathered}
$$

где

$$
\begin{aligned}
\omega_{q}(f)= & \frac{\left(p_{1},-k+q\right)+\left(p_{2}, k\right)}{m} \pm \frac{1}{V} \sum_{p}\left\{\nu\left(p_{1}-\frac{k-q}{2}-p\right)+\right. \\
& \left.+\nu\left(p_{2}+\frac{k}{2}-p\right)-\nu\left(p_{2}-\frac{k}{2}-p\right)-\nu\left(p_{1}+\frac{k-q}{2}-p\right)\right\} n_{p}
\end{aligned}
$$

и

$$
\mathcal{V}_{q}\left(f \mid f^{\prime}\right)=\overline{\mathcal{V}}_{q}\left(f^{\prime} \mid f\right)=\sum_{j=1}^{6} \mathcal{V}_{q}^{(j)}\left(p_{1}, p_{2}, k \mid p_{1}^{\prime}, p_{2}^{\prime}, k^{\prime}\right) .
$$

Слагаемые $\mathcal{V}_{q}^{(j)}\left(f \mid f^{\prime}\right)$ представляют собой потенциалы, получаемыев диаграммной технике путем суммирования бесконечных последовательностей диаграмм. Решения уравнений $(2.22),(2.23)$ с помошью $T$-матрицы, удовлетворяющей уравнению

$$
T_{q, E}\left(f \mid f^{\prime}\right)=\mathcal{V}_{q}\left(f \mid f^{\prime}\right)+\mathcal{V}_{q}(f \mid \bar{f})\left(E-\omega_{q}(\bar{f})\right)^{-1} T_{q, E}\left(\bar{f} \mid f^{\prime}\right),
$$

представляются в виде

$$
\begin{aligned}
G_{q, E}^{(0)}\left(f \mid f^{\prime}\right)= & \left\{\delta_{q}(f, \bar{f})+\left(E-\omega_{q}(f)\right)^{-1} T_{q, E}(f \mid \bar{f})\right\} \times \\
& \times\left(E-\omega_{q}(\bar{f})\right)^{-1} \chi_{q, E}^{(0)}\left(\bar{f} \mid f^{\prime}\right), \\
\chi_{q}^{(0)}\left(f \mid f^{\prime}\right)= & \eta_{q}[f \mid \bar{f}] \omega_{q}^{-1}(\bar{f})\left\{\delta_{q}\left(\bar{f}, f^{\prime}\right)-\bar{T}_{q, 0}\left(\bar{f} \mid f^{\prime}\right) \omega_{q}^{-1}\left(f^{\prime}\right)\right\},
\end{aligned}
$$

где $\bar{T}_{q, E}\left(f \mid f^{\prime}\right)=T_{q, E}\left(f^{\prime} \mid f\right)$. В результате в оператор столкновений уравнения (2.10) войдет перенормированный потенциал

$$
\nu_{E}(p \mid f)=\nu(p \mid \bar{f})\left\{\delta_{q}(\bar{f}, f)-\left(E-\omega_{q}(\bar{f})\right)^{-1} T_{q, E}(\bar{f} \mid f)\right\},
$$

в котором следует положить $E=i \varepsilon$. Уравнение, аналогичное уравнению (2.22), использовалось в работе [5] при выводе кинетических уравнений для систем со взаимодействием типа твердых сфер и с кулоновским взаимодействием. 
Полученные в этом разделе соотношения имеют достаточно общий характер, т.к. при их выводе практически не использовалась специфика задачи, принятой в качестве примера. Они могут быть применены для широкого класса физических систем. Следует, однако, отметить, что в случае сильно неидеальных систем, когда надо учитывать не только кинетический, но и потенциальный вклад в оператор плотности энергии (см. (2.21)), уравнения $(2.10),(2.11)$ не очень удобны, т.к., например, при построении уравнений гидродинамики надо учитывать уравнения для функций $G_{q, E}\left(p \mid p^{\prime}\right)$ и $G_{q, E}\left(f \mid f^{\prime}\right)$, принадлежашие различным звеньям цепочки. Поэтому, не останавливаясь на подробном исследовании уравнений $(2.10),(2.11)$ и (2.16), (2.17), перейдем к рассмотрению более эффективного способа решения задачи, основанного на бесконечной цепочке, определяющей уравнения молекулярной гидродинамики [2]. Полученные же выше формулы потребуются нам в дальнейшем.

\section{3. ЦЕПОЧКА УРАВНЕНИЙ ДЛЯ ОБОБШЕННОЙ (МОЛЕКУЛЯРНОЙ) ГИДРОДИНАМИКИ}

Примем в качестве базисных операторы плотностей сохраняющихся величин $\rho_{q}, J_{q}^{\alpha}$ и $h_{q}(2.21)$, ортогонализовав их относительно скалярных произведений (1.6), что приводит к замене $h_{q}$ на $\theta_{q}=h_{q}-\left(h_{q} \mid \rho_{-q}\right)\left(\rho_{q} \mid \rho_{-q}\right)^{-1} \rho_{q}$. В результате будем иметь столбец $A(1)$ и сопряженную строку $A^{+}\left(1^{\prime}\right)$ вида

$$
\begin{aligned}
A(1) \equiv A_{q} & =\operatorname{col}\left(\rho_{q}, J_{q}^{\alpha}, \theta_{q}\right), \\
A^{+}\left(1^{\prime}\right) & \equiv A_{q}^{+}=\left(\rho_{-q}, J_{-q}^{\beta}, \theta_{-q}\right) \quad(\alpha, \beta=x, y, z) .
\end{aligned}
$$

Для матрицы $G_{q, E}=\left(\left(A_{q} \mid A_{q}^{+}\right)\right)_{E}$, построенной по правилу умножения "строка на столбец", будем иметь согласно уравнению (1.3) при $n=1$ матричное уравнение

$$
E G_{q, E}=\chi_{q}+\left\{\eta_{q}+\left(\left(i \dot{A}_{q} \mid-i \dot{A}_{q}^{+}\right)\right)_{1, E}\right\} \chi_{q}^{-1} G_{q, E},
$$

где $\eta_{q}=\left\langle\left[A_{q}, A_{q}^{+}\right]_{-}\right\rangle=\left(i \dot{A}_{q} \mid A_{q}^{+}\right), \chi_{q}=\left(A_{q} \mid A_{q}^{+}\right)=\operatorname{diag}\left(\chi_{q}^{\rho}, \chi_{q}(\alpha \mid \beta), \chi_{q}^{\theta}\right)$ - диагональная матрица (вследствие ортогональности операторов в (3.1)), и согласно (1.5) матрица коэффициентов равна

$$
\left(\left(i \dot{A}_{q} \mid-i \dot{A}_{q}^{+}\right)\right)_{1, E}=\left(\left(i \dot{A}_{q} \mid-i \dot{A}_{q}^{+}\right)\right)_{E}-\left(\left(i \dot{A}_{q} \mid A_{q}^{+}\right)\right)_{E} G_{q, E}^{-1}\left(\left(A_{q} \mid-i \dot{A}_{q}^{+}\right)\right)_{E}
$$

Восприимчивости $\chi_{q}^{\rho}, \chi_{q}(\alpha \mid \beta), \chi_{q}^{\theta}$ и отличные от нуля компоненты матрицы $\eta_{q}=$ $\left\langle\left[A_{q}, A_{q}^{+}\right]_{-}\right\rangle$имеют вид

$$
\begin{gathered}
\chi_{q}^{\rho}=\left(\rho_{q} \mid \rho_{-q}\right), \\
\chi_{q}^{\theta}=\left(\theta_{q} \mid \theta_{-q}\right)=\left(h_{q} \mid h_{-q}\right)-\left(h_{q} \mid \rho_{-q}\right)\left(\chi_{q}^{\rho}\right)^{-1}\left(\rho_{q} \mid h_{-q}\right), \\
\chi_{q}(\alpha \mid \beta)=\left(J_{q}^{\alpha} \mid J_{-q}^{\beta}\right)=\frac{q_{\alpha} q_{\beta}}{q^{2}} \chi_{q}^{\|}+\left(\delta_{\alpha, \beta}-\frac{q_{\alpha} q_{\beta}}{q^{2}}\right) \chi_{q}^{\perp},
\end{gathered}
$$


где

$$
J_{q}^{\alpha}=\frac{q_{\alpha}}{q^{2}} i \dot{\rho}_{q}+J_{q}^{\perp \alpha}, \quad i \dot{\rho}_{q}=\left(q, J_{q}\right)=\frac{1}{\sqrt{N}} \sum_{p} \frac{(\mathbf{p}, \mathbf{q})}{m} a_{q}(p)
$$

и, следовательно,

$$
\chi_{q}^{\|}=\frac{1}{q^{2}}\left(i \dot{\rho}_{q} \mid-i \dot{\rho}_{q}\right)=\frac{1}{q^{2}}\left\langle\left[i \dot{\rho}_{q}, \rho_{-q}\right]\right\rangle=\frac{1}{m}, \quad \chi_{q}^{\perp}=\frac{1}{2}\left(\mathbf{J}_{q}^{\perp} \cdot \mid \mathbf{J}_{-q}^{\perp}\right) \cong \frac{1}{m}
$$

так что

$$
\chi_{q}^{-1}(\alpha \mid \beta)=\frac{q_{\alpha} q_{\beta}}{q^{2}} m+\left(\delta_{\alpha, \beta}-\frac{q_{\alpha} q_{\beta}}{q^{2}}\right)\left(\chi_{q}^{\perp}\right)^{-1}
$$

и

$$
\begin{aligned}
& \left\langle\left[J_{q}^{\alpha}, \rho_{-q}\right]\right\rangle=\left\langle\left[\rho_{q}, J_{-q}^{\alpha}\right]\right\rangle=\frac{q_{\alpha}}{q^{2}}\left\langle\left[i \dot{\rho}_{q}, \rho_{-q}\right]\right\rangle=\frac{q_{\alpha}}{m}, \\
& \left\langle\left[\theta_{q}, J_{-q}^{\alpha}\right]\right\rangle=\left\langle\left[J_{q}^{\alpha}, \theta_{-q}\right]\right\rangle=\frac{q_{\alpha}}{q^{2}}\left\langle\left[i \dot{\rho}_{q}, \theta_{-q}\right]\right\rangle=\frac{q_{\alpha}}{m} \eta_{q}^{\theta \ddot{\rho}}=\frac{q_{\alpha}}{m} \eta_{q}^{\ddot{\rho} \theta} .
\end{aligned}
$$

В последнем равенстве введено обозначение, выделяюшее главную зависимость $\left\langle\left[i \dot{\rho}_{q}, \theta_{-q}\right]_{-}\right\rangle$от $q$ при $q \rightarrow 0$.

Запишем уравнение (3.2) в виде системы уравнений, учитывая, что вследствие неприводимости компоненты матрицы (3.3), содержащие оператор $i \dot{\rho}_{q}$, равны нулю, т.е. $\left(\left(i \dot{\rho}_{q} \mid-i \dot{A}_{q}^{+}\right)\right)_{1, E}=\left(\left(i \dot{A}_{q} \mid-i \dot{\rho}_{-q}\right)\right)_{1, E}=0$. Имеем

$$
\begin{aligned}
E\left(\left(\rho_{q} \mid A_{q}^{+}\right)\right)_{E}= & \left(\rho_{q} \mid A_{q}^{+}\right)+\left(\left(i \dot{\rho}_{q} \mid A_{q}^{+}\right)\right)_{E} \\
E\left(\left(J_{q}^{\alpha} \mid A_{q}^{+}\right)\right)_{E}= & \left(J_{q}^{\alpha} \mid A_{q}^{+}\right)+\frac{q_{\alpha}}{m}\left(\chi_{q}^{\rho}\right)^{-1}\left(\left(\rho_{q} \mid A_{q}^{+}\right)\right)_{E}+ \\
& +\frac{q^{2}}{m} D_{q}^{\alpha, \bar{\alpha}}(E)\left(\left(J_{q}^{\bar{\alpha}} \mid A_{q}^{+}\right)\right)_{E}^{+} \\
& +\frac{q_{\alpha}}{m}\left\{\eta_{q}^{\dot{\rho} \theta}+\frac{q^{2}}{m} D_{q}^{\rho \theta}(E)\right\}\left(\chi_{q}^{\theta}\right)^{-1}\left(\left(\theta_{q} \mid A_{q}^{+}\right)\right)_{E} \\
E\left(\left(\theta_{q} \mid A_{q}^{+}\right)\right)_{E}= & \left(\theta_{q} \mid A_{q}^{+}\right)+\left\{\eta_{q}^{\theta \dot{\rho}}+\frac{q^{2}}{m} D_{q}^{\theta \rho}(E)\right\}\left(\left(i \dot{\rho}_{q} \mid A_{q}^{+}\right)\right)_{E}+ \\
& +\frac{q^{2}}{m} D_{q}^{\theta \theta}(E)\left(\left(\theta_{q} \mid A_{q}^{+}\right)\right)_{E}
\end{aligned}
$$

где во втором уравнении черта над $\bar{\alpha}$ означает суммирование по $x, y, z$ и

$$
D_{q}^{\alpha, \beta}(E)=\frac{m}{q^{2}}\left(\left(i \dot{J}_{q}^{\alpha} \mid-i \dot{J}_{-q}^{\beta}\right)\right)_{1, E}=\frac{q_{\alpha} q_{\beta}}{q^{2}} D_{q}^{\rho \rho}(E)+\left(\delta_{\alpha, \beta}-\frac{q_{\alpha} q_{\beta}}{q^{2}}\right) D_{q}^{\perp}(E) .
$$

Обобщенные кинетические коэффициенты записываются в виде

$$
\begin{aligned}
& D_{q}^{\rho \rho}(E)=\left(\frac{m}{q^{2}}\right)^{2}\left(\left(i^{2} \ddot{\rho}_{q} \mid i^{2} \ddot{\rho}_{-q}\right)\right)_{1, E}, \quad D_{q}^{\rho \theta}(E)=\left(\frac{m}{q^{2}}\right)^{2}\left(\left(i^{2} \ddot{\rho}_{q} \mid-i \dot{\theta}_{-q}\right)\right)_{1, E}, \\
& D_{q}^{\theta \rho}(E)=\left(\frac{m}{q^{2}}\right)^{2}\left(\left(i \dot{\theta}_{q} \mid i^{2} \ddot{\rho}_{-q}\right)\right)_{1, E}, \quad D_{q}^{\theta \theta}(E)=\frac{m}{q^{2}}\left(\left(i \dot{\theta}_{q} \mid-i \dot{\theta}_{-q}\right)\right)_{1, E}\left(\chi_{q}^{\theta}\right)^{-1}
\end{aligned}
$$


и

$$
D_{q}^{\perp}(E)=\frac{1}{2 \chi_{q}^{\perp}} \frac{m}{q^{2}}\left(\left(i \dot{\mathbf{J}}_{q}^{\perp} \cdot \mid-i \dot{\mathbf{J}}_{-q}^{\perp}\right)\right)_{1, E},
$$

где $i^{2} \ddot{\rho}_{q}=\left(q, i \dot{J}_{-q}\right)=\left[\left[\rho_{q}, \mathcal{H}\right]_{-}, \mathcal{H}\right]_{-}$. Уравнения $(3.8)$ представляют собой линеаризованные уравнения молекулярной гидродинамики [2]. Выражения для кинетических коэффициентов (3.10), (3.11) справедливы (в отличие от обычной гидродинамики) при всех значениях $E$ и $q$. Они связаны с обычными кинетическими коэффициентами соотношениями

$$
\begin{gathered}
\mathcal{D}_{l}=\frac{\zeta+\frac{4}{3} \eta}{m n}=\frac{i}{m} D_{q=0}^{\rho \rho} \quad(E=i \varepsilon), \quad \mathcal{D}^{\theta}=\frac{\varkappa}{c_{v}}=\frac{i}{m} D_{q=0}^{\theta \theta} \quad(E=i \varepsilon), \\
\nu=\frac{\eta}{m n}=\frac{i}{m} D_{q=0}^{\perp} \quad(E=i \varepsilon),
\end{gathered}
$$

где $n=N / V, \varepsilon \rightarrow 0$.

Вычисление обобщенных кинетических коэффициентов (3.10), (3.11). Для этого воспользуемся уравнениями, полученными из цепочки (1.3), если $n=2,3, \ldots$. При этом возможны два пути. На первом из них в качестве следующих за (3.8) звеньев можно принять уравнения для неприводимых относительно (3.1) функций, построенных на операторах $a(2)=a_{q}(p)$ и $a(3)=a_{q}(f)(2.2)$, через которые выражаются кинетические коэффициенты $(3.10),(3.11)$. Другой путь, обладающий существенными преимуществами по сравнению с первым, основан на использовании в качестве второго звена уравнения для самих кинетических коэффициентов. В этом случае оператор $a(2)$ представляет собой столбец из операторов, т.е. $a(2) \equiv a_{q}^{\prime}=\operatorname{col}\left(i \dot{j}_{q}^{\alpha}, i \dot{h}_{q}\right)$ и

$$
\begin{aligned}
i \dot{j}_{q}^{\alpha}=\left[J_{q}^{\alpha}, \mathcal{H}\right]_{-}= & \frac{1}{\sqrt{N}} \sum_{p} \frac{p_{\alpha}}{m} \frac{(\mathbf{p}, \mathbf{q})}{m} a_{q}(p)+ \\
& +\frac{1}{\sqrt{N} V} \sum_{f=\left(p_{1}, p_{2}, k\right)} \nu_{q}^{\alpha}(f) a_{q}(f), \\
i \dot{h}_{q}=\left[h_{q}, \mathcal{H}\right]_{-}= & \frac{1}{\sqrt{N}} \sum_{p}\left(\frac{p^{2}}{2 m}-\frac{q^{2}}{8 m}\right) \frac{(\mathbf{p}, \mathbf{q})}{m} a_{q}(p)+ \\
& +\frac{1}{\sqrt{N} V} \sum_{f=\left(p_{1}, p_{2}, k\right)} \nu_{q}^{h}(f) a_{q}(f),
\end{aligned}
$$

где

$$
\begin{aligned}
\nu_{q}^{\alpha}(f) & =\frac{k_{\alpha}}{2 m} \nu(k)+\frac{-k_{\alpha}+q_{\alpha}}{2 m} \nu(-k+q), \\
\nu_{q}^{h}(f) & =\frac{1}{2}(\nu(k)+\nu(-k+q)) \frac{\left(\mathbf{p}_{1}, \mathbf{q}\right)}{m}+\frac{1}{2}(\nu(k)-\nu(-k+q)) \frac{\left(\mathbf{p}_{1}, \mathbf{k}\right)}{m}
\end{aligned}
$$

(см. $(2.21),(2.1))$. 
Ортогонализуя относительно (3.1) операторы (3.13), (3.14), получим

$$
A(2) \equiv A_{q}^{\prime}=\operatorname{col}\left(J_{q}^{\prime \alpha}, \theta_{q}^{\prime}\right), \quad A^{+}\left(2^{\prime}\right) \equiv A_{q}^{\prime+}=\left(-J_{-q}^{\prime \beta},-\theta_{-q}^{\prime}\right)
$$

где с учетом соотношений (3.4)-(3.7)

$$
\begin{aligned}
J_{q}^{\prime \alpha} & =i \dot{J}_{q}^{\alpha}-\left(i \dot{J}_{q}^{\alpha} \mid A_{q}^{+}\right) \chi_{q}^{-1} A_{q}=i \dot{J}_{q}^{\alpha}-\frac{q_{\alpha}}{m}\left(\chi_{q}^{\rho}\right)^{-1} \rho_{q}-\frac{q_{\alpha}}{m} \eta_{q}^{\dot{\rho} \theta}\left(\chi_{q}^{\theta}\right)^{-1} \theta_{q} \\
\theta_{q}^{\prime} & =i \dot{\theta}_{q}-\left(i \dot{\theta}_{q} \mid A_{q}^{+}\right) \chi_{q}^{-1} A_{q}=i \dot{\theta}_{q}-\eta_{q}^{\theta} \dot{\rho}_{\dot{\rho}_{q}} .
\end{aligned}
$$

Для матричной, неприводимой относительно $A_{q}$ функции Грина $G_{E}\left(2 \mid 2^{\prime}\right) \equiv G_{q, E}^{\prime}=$ $\left(\left(A_{q}^{\prime} \mid A_{q}^{\prime+}\right)\right)_{1, E}=\left(\left(a_{q}^{\prime} \mid a_{q}^{\prime+}\right)\right)_{1, E}$ будем иметь следуюшее уравнение:

$$
E G_{q, E}^{\prime}=\chi_{q}^{\prime}+\left\{\eta_{q}^{\prime}+\left(\left(i \dot{A}_{q}^{\prime} \mid-i \dot{A}_{q}^{\prime+}\right)\right)_{2, E}\right\} \chi_{q}^{\prime-1} G_{q, E}^{\prime}
$$

где

$$
\chi_{q}^{\prime}=\operatorname{diag}\left(\left(\frac{q}{m}\right)^{2} \chi_{q}^{\prime}(\alpha \mid \beta), \frac{q^{2}}{m} \chi_{q}^{\theta^{\prime}}\right)
$$

$$
\begin{aligned}
\chi_{q}^{\prime}(\alpha \mid \beta) & =\left(\frac{m}{q}\right)^{2}\left(J_{q}^{\prime \alpha} \mid-J_{-q}^{\prime \beta}\right)=\frac{q_{\alpha} q_{\beta}}{q^{2}} \chi_{q}^{\rho^{\prime \prime}}+\left(\delta_{\alpha, \beta}-\frac{q_{\alpha} q_{\beta}}{q^{2}}\right) \chi_{q}^{\prime \perp} \\
\chi_{q}^{\theta^{\prime}} & =\frac{m}{q^{2}}\left(\theta_{q}^{\prime} \mid-\theta_{-q}^{\prime}\right)=\frac{m}{q^{2}}\left\langle\left[i \dot{\theta}_{q}, \theta_{-q}\right]_{-}\right\rangle-\eta_{q}^{\theta \dot{\rho}} \eta_{q}^{\dot{\rho} \theta} \\
\chi_{q}^{\rho^{\prime \prime}} & =\left(\frac{m}{q^{2}}\right)^{2}\left(\rho_{q}^{\prime \prime} \mid \rho_{-q}^{\prime \prime}\right)= \\
& =\left(\frac{m}{q^{2}}\right)^{2}\left\langle\left[i^{2} \ddot{\rho}_{q},-i \dot{\rho}_{-q}\right]_{-}\right\rangle-\left(\chi_{q}^{\rho}\right)^{-1}-\eta_{q}^{\dot{\rho} \theta}\left(\chi_{q}^{\theta}\right)^{-1} \eta_{q}^{\theta \dot{\rho}}
\end{aligned}
$$

здесь

$$
\rho_{q}^{\prime \prime}=\left(\mathbf{q}, \mathbf{J}_{q}^{\prime \alpha}\right)=i^{2} \ddot{\rho}_{q}-\frac{q^{2}}{m}\left(\chi_{q}^{\rho}\right)^{-1} \rho_{q}-\frac{q^{2}}{m} \eta_{q}^{\dot{\rho} \theta}\left(\chi_{q}^{\theta}\right)^{-1} \eta_{q}^{\theta \dot{\rho}}
$$

Отличные от нуля компоненты матрицы $\eta_{q}^{\prime}=\left\langle\left[A_{q}^{\prime}, A_{q}^{\prime+}\right]_{-}\right\rangle$имеют вид

$$
\begin{gathered}
\left\langle\left[J_{q}^{\prime \alpha},-\theta_{-q}^{\prime}\right]_{-}\right\rangle=\frac{q_{\alpha}}{m} \frac{q^{2}}{m} \eta_{q}^{\rho^{\prime \prime} \theta^{\prime}}, \quad\left\langle\left[\theta_{q}^{\prime},-J_{-q}^{\prime \beta}\right]_{-}\right\rangle=\frac{q_{\beta}}{m} \frac{q^{2}}{m} \eta_{q}^{\theta^{\prime} \rho^{\prime \prime}}, \\
\eta_{q}^{\rho^{\prime \prime} \theta^{\prime}}=\eta_{q}^{\theta^{\prime} \rho^{\prime \prime}}=\left(\frac{m}{q^{2}}\right)^{2}\left\langle\left[\theta_{q}^{\prime}, \rho_{-q}^{\prime \prime}\right]_{-}\right\rangle .
\end{gathered}
$$

Матричная функция $\left(\left(i \dot{A}_{q}^{\prime} \mid-i \dot{A}_{q}^{\prime+}\right)\right)_{2, E}=\left(\left(i \dot{a}_{q} \mid-i \dot{a}_{q}^{+}\right)\right)_{2, E}$ неприводима относительно операторов $A(1)=A_{q}$ и $A(2)=A_{q}^{\prime}$. 
Выбрав систему координат с осью $z$, направленной вдоль вектора q, запишем матричное уравнение (3.17) в виде системы уравнений. Для поперечных компонент потока имеем

$$
\begin{gathered}
\left\{E-\left(\frac{m}{q^{2}}\right)^{2}\left(\left(i^{2} \ddot{J}_{q}^{x} \mid i^{2} \ddot{J}_{-q}^{x}\right)\right)_{2, E}\left(\chi_{q}^{\prime \perp}\right)^{-1}\right\}\left(\left(J_{q}^{\prime x} \mid-J_{-q}^{\prime x}\right)\right)_{1, E}= \\
=\left(J_{q}^{\prime x} \mid-J_{-q}^{\prime x}\right)=\left(\frac{q}{m}\right)^{2} \chi_{q}^{\prime \perp}
\end{gathered}
$$

для продольных компонент -

$$
\begin{aligned}
& \left\{E-\left(\frac{m}{q^{2}}\right)^{2}\left(\left(i^{3 \dddot{\rho}_{q}} \mid-i^{3} \dddot{\rho}_{-q}\right)\right)_{2, E}\left(\chi_{q}^{\rho^{\prime \prime}}\right)^{-1}\right\}\left(\left(\rho_{q}^{\prime \prime} \mid A_{q}^{\prime+}\right)\right)_{1, E}- \\
& -\frac{q^{2}}{m}\left\{\eta_{q}^{\rho^{\prime \prime} \theta^{\prime}}+\left(\frac{m}{q^{2}}\right)^{2}\left(\left(i^{3} \dddot{\rho}_{q} \mid i^{2} \ddot{h}_{-q}\right)\right)_{2, E}\right\}\left(\chi_{q}^{\theta^{\prime}}\right)^{-1}\left(\left(\theta_{q}^{\prime} \mid A_{q}^{\prime+}\right)\right)_{1, E}=\left(\rho_{q}^{\prime \prime} \mid A_{q}^{\prime+}\right), \\
& -\left\{\eta_{q}^{\theta^{\prime} \rho^{\prime \prime}}+\left(\frac{m}{q^{2}}\right)^{2}\left(\left(i^{2} \ddot{h}_{q} \mid-i^{3} \dddot{\rho}_{-q}\right)\right)_{2, E}\right\}\left(\chi_{q}^{\rho^{\prime \prime}}\right)^{-1}\left(\left(\rho_{q}^{\prime \prime} \mid A_{q}^{\prime+}\right)\right)_{1, E}+ \\
& \quad+\left\{E-\frac{m}{q^{2}}\left(\left(i^{2} \ddot{h}_{q} \mid i^{2} \ddot{h}_{-q}\right)\right)_{2, E}\left(\chi_{q}^{\theta^{\prime}}\right)^{-1}\right\}\left(\left(\theta_{q}^{\prime} \mid A_{q}^{\prime+}\right)\right)_{1, E}=\left(\theta_{q}^{\prime} \mid A_{q}^{\prime+}\right),
\end{aligned}
$$

где $A_{q}^{\prime+}=\rho_{-q}^{\prime \prime}, \theta_{-q}^{\prime}($ см. $(3.15))$.

Выражения для операторов

$$
i^{2} \ddot{J}_{q}^{\alpha}=\left[i \dot{J}_{q}^{\alpha}, \mathcal{H}\right]_{-} \quad\left(i^{3} \dddot{\rho}_{q}=q_{\bar{\alpha}} i^{2} \ddot{J}_{q}^{\bar{\alpha}}\right) \quad \text { и } i^{2} \ddot{h}_{q}=\left[i \dot{h}_{q}, \mathcal{H}\right]_{-},
$$

получаемые из (3.13), (3.14) с помошью уравнения движения (2.1), имеют (в отличие от (3.13), (3.14)) в правых частях в первых членах дополнительный множитель $(\mathbf{p}, \mathbf{q}) / m$, а функции $\nu_{q}^{\alpha}(f)$ и $\nu_{q}^{h}(f)$ заменяются соответственно на

$$
\begin{aligned}
\nu_{q}^{\prime \alpha}(f)= & \nu(k)\left(\frac{p_{1, \alpha}}{m} \frac{(\mathbf{k}, \mathbf{q})}{m}+\frac{k_{\alpha}}{m} \frac{\left(\mathbf{p}_{1}, \mathbf{q}\right)}{m}\right)+ \\
& +\left(\nu(k) \frac{k_{\alpha}}{m}+\nu(-k+q) \frac{-k_{\alpha}+q_{\alpha}}{m}\right) \frac{\left(\mathbf{p}_{1},-\mathbf{k}+\mathbf{q}\right)}{m}
\end{aligned}
$$

и

$$
\begin{aligned}
\nu_{q}^{\prime h}(f)= & \nu(k)\left\{\frac{\left(\mathbf{p}_{1}, \mathbf{k}\right)}{m} \frac{\left(\mathbf{p}_{1}, \mathbf{q}\right)}{m}+\left(\frac{p_{1}^{2}}{2 m}+\frac{k^{2}-q^{2}}{8 m}\right) \frac{(\mathbf{k}, \mathbf{q})}{m}\right\}+ \\
& +\left\{\frac{\nu(k)-\nu(-k+q)}{2} \frac{\left(\mathbf{p}_{1}, \mathbf{k}\right)}{m}+\frac{\nu(k)+\nu(-k+q)}{2} \frac{\left(\mathbf{p}_{1}, \mathbf{q}\right)}{m}\right\} \times \\
& \times\left\{\frac{\left(\mathbf{p}_{1},-\mathbf{k}+\mathbf{q}\right)}{m}+\frac{\left(\mathbf{p}_{2}, \mathbf{k}\right)}{m}\right\}
\end{aligned}
$$


Кроме того, в оператор $i^{2} \ddot{h}_{q}$ войдет член с $a_{q}(g)$ (см. (2.2)), вклад которого, однако, несушествен в гидродинамическом пределе. Таким образом, как и на упомянутом выше первом пути, мы снова приходим к необходимости вычисления функций Грина, построенных на операторах $a_{q}(p)$ и $a_{q}(f)$, но уже входящих не в сами коэффициенты переноса (3.10), (3.11), а в коэффициенты уравнений для них (см. (3.22), (3.23)). Преимушество второго пути состоит в том, что операторы $i^{2} \ddot{J}_{q}^{x}, i^{3} \dddot{\rho}_{q}, i^{2} \ddot{h}_{q}$ в отличие от операторов $i \dot{j}_{q}^{x}, i^{2} \ddot{\rho}_{q}, i \dot{h}_{q}$ уже не являются производными квазиинтегралов движения. Поэтому при $q \rightarrow 0$ вклад в коэффициенты уравнений (3.22), (3.23) функций, описываюших свободное движение, вьпадает. При непосредственном вычислении надо было бы учитывать оба члена в правых частях уравнений $(3.13),(3.14)$, имеюших различную операторную структуру, т.к. они одинаково зависят от импульса $q$ при $q \rightarrow 0$. Это обстоятельство привело бы к существенному усложнению вычислений.

Подставляя в первое уравнение системы (3.23) $A_{q}^{\prime+}=\rho_{q}^{\prime \prime}$, а во второе $-A_{q}^{\prime+}=-\theta_{-q}^{\prime}$, убеждаемся (учитывая различную четность операторов $i^{3} \dddot{\rho}_{q}$ и $i^{2} \ddot{h}_{q}$ ), что при $q \rightarrow 0$ в этих уравнениях (в левой их части) можно пренебречь соответственно вторым и первым членами. В результате получим (см. (3.10), (3.11))

$$
\begin{aligned}
D_{q}^{\rho \rho}(E) & =\left(\frac{m}{q^{2}}\right)^{2}\left(\left(i^{2} \ddot{\rho}_{q} \mid i^{2} \ddot{\rho}_{-q}\right)\right)_{1, E} \approx \\
& \approx \frac{\chi_{q}^{\rho^{\prime \prime}}}{E-\left(\frac{m}{q^{2}}\right)^{2}\left(\left(i^{3} \dddot{\rho}_{q} \mid-i^{3} \dddot{\rho}_{-q}\right)\right)_{2, E}\left(\chi_{q}^{\rho^{\prime \prime}}\right)^{-1}} \approx-i \chi^{\rho^{\prime \prime}} \tau, \\
D_{q}^{\theta \theta}(E) & =\frac{m}{q^{2}}\left(\left(i \dot{h}_{q} \mid-i \dot{h}_{-q}\right)\right)_{1, E}\left(\chi_{q}^{\theta}\right)^{-1} \approx \\
& \approx \frac{\chi_{q}^{\theta^{\prime}} / \chi_{q}^{\theta}}{E-\frac{m}{q^{2}}\left(\left(i^{2} \ddot{h}_{q} \mid i^{2} \ddot{h}_{-q}\right)\right)_{2, E}\left(\chi_{q}^{\theta^{\prime}}\right)^{-1}} \approx-i \frac{\chi^{\theta^{\prime}}}{\chi^{\theta}} \tau^{\prime}
\end{aligned}
$$

и согласно $(3.22)$

$$
\begin{aligned}
D_{q}^{\perp}(E) & =\left(\frac{m}{q}\right)^{2}\left(\left(J_{q}^{\prime x} \mid-J_{-q}^{\prime x}\right)\right)_{1, E}\left(m \chi_{q}^{\perp}\right)^{-1}= \\
& =\frac{\chi_{q}^{\perp} / m \chi_{q}^{\perp}}{E-\left(\frac{m}{q}\right)^{2}\left(\left(i^{2} \ddot{J}_{q}^{x} \mid i^{2} \ddot{J}_{-q}^{x}\right)\right)_{2, E}\left(\chi_{q}^{\perp \perp}\right)^{-1}} \approx-i \frac{\chi^{\perp \perp}}{m \chi^{\perp}} \tau^{\prime \prime} .
\end{aligned}
$$

Последние приближенные равенства в правых частях соотношений (3.24)-(3.26) представляют собой предельные значения при $q \rightarrow 0, E=i \varepsilon \rightarrow 0$ и являются, по существу, определениями времен релаксации $\tau, \tau^{\prime}$ и $\tau^{\prime \prime}$. Для "перекрестных" кинетических коэффициентов с учетом равенств $(3.24),(3.25)$ имеем при $q \rightarrow 0$

$$
D_{q}^{\rho \theta}(E) \cong-\left\{\eta_{q}^{\rho^{\prime \prime} \theta^{\prime}}+\left(\frac{m}{q^{2}}\right)^{2}\left(\left(i^{3} \dddot{\rho}_{q} \mid i^{2} \ddot{h}_{-q}\right)\right)_{2, E=i \varepsilon}\right\} \tau \tau^{\prime}
$$


и аналогичное выражение для $D_{q}^{\theta \rho}(E)$.

Времена релаксации, определяемые соотношениями (3.24)-(3.27), выражаются через неприводимую относительно $A_{q}$ и $A_{q}^{\prime}$ функцию Грина $\left(\left(a_{q}(f) \mid a_{q}^{+}\left(f^{\prime}\right)\right)\right)_{2, E}$, т.к. вклады свободного движения по указанной выше причине в пределе $q \rightarrow 0, E \rightarrow 0$ выпадают. Так, например, для величины $1 / \tau^{\prime \prime}$, исходя из соотношения $(3.26)$, будем иметь (полагаем $\nu(k) \cong U$ )

$$
\begin{aligned}
\frac{1}{\tau^{\prime \prime}}= & i \lim _{q \rightarrow 0} \frac{1}{\chi_{q}^{\perp}} \frac{m}{q^{2}}\left(\left(i^{2} \ddot{J}_{q}^{x} \mid i^{2} \ddot{J}_{-q}^{x}\right)\right)_{2, E}= \\
= & i \lim _{q \rightarrow 0} \frac{1}{\chi_{q}^{\perp}} \frac{U^{2}}{N V^{2}} \sum_{f, f^{\prime}} \frac{p_{1 x} k_{z}+k_{x} p_{1 z}}{m} \times \\
& \times \frac{p_{1 x}^{\prime} k_{z}^{\prime}+k_{x}^{\prime} p_{1, z}^{\prime}}{m}\left(\left(a_{q}(f) \mid a_{q}^{+}\left(f^{\prime}\right)\right)\right)_{2, E}
\end{aligned}
$$

Входящие в коэффищиенты уравнений (3.8), (3.22), (3.23) статические корреляционные функции выражаются через функции $\left(a_{q}(p) \mid a_{q}^{+}\left(p^{\prime}\right)\right), \quad\left(a_{q}(p) \mid a_{q}^{+}\left(f^{\prime}\right)\right)$, $\left(a_{q}(f) \mid a_{q}^{+}\left(f^{\prime}\right)\right)$ и могут быть найдены из цепочки уравнений $(2.16),(2.17)$.

Уравнения (3.8), (3.22), (3.23), являюшиеся точными, были получены в работе [3]. В работе [4] эти уравнения подробно исследованы в случае слабо неидеального газа. При этом кинетические коэффициенты (3.12), получаемые путем прямого вычисления предельных значений корреляционных функций в знаменателях выражений $(3.24),(3.26)$ в приближении идеального газа, совпадают с результатами кинетической теории, основанной на решении уравнения Больцмана методом Чепмена-Энскога [6]. Для самих же корреляционных функций получаются выражения, пригодные как в гидродинамической области значений $E$ и $q$, так и вне ее.

В статье [7] был использован близкий к работе [3] метод численного расчета временных корреляционных функций классической системы со взаимодействием ЛеннардДжонса между частицами для отдельных приведенных значений волнового вектора и времени. В рамках метода Мори в работе [7] использовалась система уравнений для компонент матрицы размерности $5 \times 5$, что эквивалентно применению базисного столбца, включающего как $A_{q}(3.1)$, так и $A_{q}^{\prime}(3.15)$.

Последующие разделы данной работы посвящены разработке методов построения цепочки уравнений для функций возрастаюшей степени неприводимости, когда в качестве исходных приняты уравнения молекулярной гидродинамики (3.8), (3.22), (3.23), а также построению на основе такой цепочки функций распределения, пригодных для вычисления различных моментов, в том числе и отличных от гидродинамических. Таким путем мы достигаем полноты описания исследуемой системы, присушей кинетическому уравнению.

\section{4. ТРЕТЬЕ И ЧЕТВЕРТОЕ УРАВНЕНИЯ ЦЕПОЧКИ}

В качестве следующих за (3.2) и (3.17) звеньев цепочки примем уравнения для функций, построенных на операторах (2.2), через которые выражаются коэффициенты урав- 
нений (3.22)-(3.26), определяющих кинетические коэффициенты. Только теперь это будут операторы третьего и последующих звеньев $a(3)=a_{q}(p), a(4)=a_{q}(f), a(5)=$ $a_{q}(g), \ldots$ Построенные на них функции

$$
\begin{aligned}
& G_{E}\left(3 \mid 3^{\prime}\right)=G_{q, E}\left(p \mid p^{\prime}\right)=\left(\left(a_{q}(p) \mid a_{q}^{+}\left(p^{\prime}\right)\right)\right)_{2, E}, \\
& G_{E}\left(4 \mid 4^{\prime}\right)=G_{q, E}\left(f \mid f^{\prime}\right)=\left(\left(a_{q}(f) \mid a_{q}^{+}\left(f^{\prime}\right)\right)\right)_{3, E} \quad \text { и т.д. }
\end{aligned}
$$

будут неприводимы относительно операторов $A_{q}(3.1)$ и $A_{q}^{\prime}(3.15), \ldots$.

Уравнения для этих функций внешне схожи с уравнениями $(2.10),(2.11)$ для функций

$$
\widetilde{G}_{q, E}\left(p \mid p^{\prime}\right)=\left(\left(a_{q}(p) \mid a_{q}^{+}\left(p^{\prime}\right)\right)\right)_{E}, \quad \widetilde{G}_{q, E}\left(f \mid f^{\prime}\right)=\left(\left(a_{q}(f) \mid a_{q}^{+}\left(f^{\prime}\right)\right)\right)_{\tilde{1}, E}, \quad \ldots
$$

Последние функции, чтобы отличить их от функций (4.1), будем помечать тильдой (индекс $\tilde{1}$ означает в этом случае неприводимость относительно оператора $\left.a_{q}(p)\right)$. Уравнения для функций (4.1) обладают сушественными дополнительными свойствами, обусловленными неприводимостью.

Уравнение для функции $G_{q, E}\left(p \mid p^{\prime}\right)$. В соответствии с принятой схемой вычислений ортогонализуем оператор $a(3)=a_{q}(p)=a_{p-\frac{q}{2}}^{+} a_{p+\frac{q}{2}}$ относительно столбцов $A_{q}(3.1)$ и $A_{q}^{\prime}(3.15)$, положив

$$
\begin{aligned}
A(3) & \equiv A_{q}(p)=a_{q}(p)-\left(a_{q}(p) \mid A_{q}^{+}\right) \chi_{q}^{-1} A_{q}-\left(a_{q}(p) \mid A_{q}^{\prime+}\right) \chi_{q}^{\prime-1} A_{q}^{\prime}= \\
& =a_{q}(p)-\left(a_{q}(p) \mid \tilde{A}_{q}^{+}\right) \tilde{\chi}_{q}^{-1} \tilde{A}_{q}-\left(a_{q}(p) \mid \tilde{A}_{q}^{\prime+}\right) \tilde{\chi}_{q}^{\prime-1} \tilde{A}_{q}^{\prime}= \\
& =\delta_{q}(p, \bar{p}) \tilde{A}_{q}(\bar{p}), \\
A^{+}\left(3^{\prime}\right) & \equiv A_{q}^{+}\left(p^{\prime}\right)=\tilde{A}_{q}^{+}(\bar{p}) \bar{\delta}_{q}\left(\bar{p}, p^{\prime}\right),
\end{aligned}
$$

где

$$
\tilde{\chi}_{q}=\left(\tilde{A}_{q} \mid \tilde{A}_{q}^{+}\right), \quad \tilde{\chi}_{q}^{\prime}=\left(\tilde{A}_{q}^{\prime} \mid \tilde{A}_{q}^{\prime+}\right), \quad \tilde{A}_{q}(p)=a_{q}(p)-\left(a_{q}(p) \mid \tilde{A}_{q}^{\prime+}\right) \tilde{\chi}_{q}^{\prime-1} \tilde{A}_{q}^{\prime}
$$

$$
\begin{aligned}
& \delta_{q}\left(p, p^{\prime}\right)=\delta_{p, p^{\prime}}-\left(a_{q}(p) \mid \tilde{A}_{q}^{+}\right) \tilde{\chi}_{q}^{-1}\left(\tilde{A}_{q} \mid a_{q}^{+}\left(\bar{p}^{\prime}\right)\right) \widetilde{\chi}_{q}^{-1}\left(\bar{p}^{\prime} \mid p^{\prime}\right) \\
& \bar{\delta}_{q}\left(p, p^{\prime}\right)=\delta_{p, p^{\prime}}-\tilde{\chi}_{q}^{-1}(p \mid \bar{p})\left(a_{q}(\bar{p}) \mid \tilde{A}_{q}^{+}\right) \tilde{\chi}_{q}^{-1}\left(\tilde{A}_{q} \mid a_{q}^{+}\left(p^{\prime}\right)\right)
\end{aligned}
$$

a

$$
\widetilde{\chi}_{q}\left(p \mid p^{\prime}\right)=\left(a_{q}(p) \mid a_{q}^{+}\left(p^{\prime}\right)\right) \quad\left(\text { см. } \chi_{q}\left(p \mid p^{\prime}\right)(2.9)\right)
$$

и

$$
\left(\tilde{A}_{q} \mid a_{q}^{+}\left(\bar{p}^{\prime}\right)\right) \tilde{\chi}_{q}^{-1}\left(\bar{p}^{\prime} \mid \bar{p}\right)\left(a_{q}(\bar{p}) \mid \tilde{A}_{q}^{\prime}\right)=\left(\tilde{A}_{q} \mid \tilde{A}_{q}^{\prime+}\right)=0 .
$$

Во втором равенстве формулы (4.2) произведено перераспределение операторов между $A_{q}$ и $A_{q}^{\prime}$. В столбце $\tilde{A}_{q}=\operatorname{col}\left(\rho_{q}, J_{q}^{\alpha}\right)$ оставлены операторы, выражающиеся только через оператор $a_{q}(p)$ (см. $(2.21)$ ), а оператор $\theta_{q}$ перенесен из столбца $A_{q}$ в столбец 
$\tilde{A}^{\prime}=\operatorname{col}\left(\theta_{q}, J_{q}^{\prime \alpha}, \theta_{q}^{\prime}\right)$. При этом $\widetilde{\chi}_{q}$ и $\widetilde{\chi}_{q}^{\prime}$ остались диагональными матрицами с компонентами из $\chi_{q}$ и $\chi_{q}^{\prime}$.

Оператор $A_{q}(p)(4.2)$ обладает очевидными свойствами: $\quad\left(A_{q}(p) \mid A_{q}^{+}\right)=$ $\left(A_{q}(p) \mid A_{q}^{\prime+}\right)=0$. С учетом соотношений $(2.21)$ также имеем

$$
\begin{aligned}
& \frac{1}{\sqrt{N}} \sum_{p} A_{q}(p)=\frac{1}{\sqrt{N}} \sum_{p} \frac{p_{\alpha}}{m} A_{q}(p)=0 \\
& \text { или }\left(\tilde{A}_{q} \mid a_{q}^{+}\left(\bar{p}^{\prime}\right)\right) \tilde{\chi}_{q}^{-1}\left(\bar{p}^{\prime} \mid \bar{p}\right) A_{q}(\bar{p})=0, \\
& \frac{1}{\sqrt{N}} \sum_{p} \frac{p^{2}}{2 m} A_{q}(p)=-h_{q}^{\text {int }}+\left(h_{q}^{\text {int }} \mid \tilde{A}_{q}^{+}\right) \tilde{\chi}_{q}^{-1} \tilde{A}_{q}+\left(h_{q}^{\text {int }} \mid \tilde{A}_{q}^{\prime+}\right) \tilde{\chi}_{q}^{\prime-1} \tilde{A}_{q}^{\prime} .
\end{aligned}
$$

Для выражений

$$
\frac{1}{\sqrt{N}} \sum_{p} \frac{p_{\alpha}}{m} \frac{(\mathbf{p}, \mathbf{q})}{m} A_{q}(p), \quad \frac{1}{\sqrt{N}} \sum_{p}\left(\frac{p^{2}}{2 m}-\frac{q^{2}}{8 m}\right) \frac{(\mathbf{p}, \mathbf{q})}{m} A_{q}(p)
$$

будем иметь равенства, аналогичные соотношению (4.6) с заменой $h_{q}^{\text {int }}$ соответственно на $\left(i \dot{j}_{q}^{\alpha}\right)^{\text {int }}$ и $\left(i \dot{h}_{q}\right)^{\text {int }}$, равных последним членам в правых частях равенств (3.13) и (3.14).

Аналогичными свойствами обладают функции

$$
\begin{aligned}
G_{q, E}\left(p \mid p^{\prime}\right) & =\left(\left(A_{q}(p) \mid A_{q}^{+}\left(p^{\prime}\right)\right)\right)_{2, E}=\left(\left(a_{q}(p) \mid a_{q}^{+}\left(p^{\prime}\right)\right)\right)_{2, E} \\
\chi_{q}\left(p \mid p^{\prime}\right) & =\left(A_{q}(p) \mid A_{q}^{+}\left(p^{\prime}\right)\right)=\lim _{E \rightarrow \infty} E G_{q, E}\left(p \mid p^{\prime}\right)
\end{aligned}
$$

Существенное отличие цепочки уравнений для молекулярной гидродинамики от цепочки для кинетического уравнения, рассмотренной во втором разделе, состоит в том, что вследствие условий (4.5) обратные матрицы $\chi_{q}^{-1}\left(p \mid p^{\prime}\right)$ и $G_{q, E}^{-1}\left(p \mid p^{\prime}\right)$, сушествование которых является необходимым условием для построения уравнений в рамках предлагаемой методики, в обычном смысле не сушествуют. Действительно, если бы имело место равенство $\chi_{q}\left(p \mid \bar{p}^{\prime}\right) \chi_{q}^{-1}\left(\bar{p}^{\prime} \mid p^{\prime}\right)=\delta_{p, p_{1}^{\prime}}$, то, умножив его сначала на 1 , а потом на $p_{\alpha} / m$ и суммируя, мы получили бы в соответствии с соотношением (4.5) слева нуль, а справа величину, отличную от нуля. Однако для соблюдения необходимых условий построения уравнений (см. (I.3.11)-(I.3.14)) нам достаточно, чтобы они выполнялись в пространстве, ортогональном всем предыдушим операторам. Поэтому можно определить обратную матрищу $\chi_{q}^{-1}\left(p \mid p^{\prime}\right)$ с помощью соотношений

$$
\chi_{q}\left(p \mid \bar{p}^{\prime}\right) \chi_{q}^{-1}\left(\bar{p}^{\prime} \mid p^{\prime}\right)=\delta_{q}\left(p, p^{\prime}\right), \quad \chi_{q}^{-1}(p \mid \bar{p}) \chi_{q}\left(\bar{p} \mid p^{\prime}\right)=\bar{\delta}_{q}\left(p, p^{\prime}\right) .
$$

В пространстве, ортогональном $A_{q}$ и $A_{q}^{\prime}$, символы $\delta_{q}\left(p \mid p^{\prime}\right)$ и $\bar{\delta}_{q}\left(p \mid p^{\prime}\right)(4.4)$ действуют как обычные символы Кронекера, т.е.

$$
\begin{gathered}
\delta_{q}(p, \bar{p}) \delta_{q}\left(\bar{p}, p^{\prime}\right)=\delta_{p, \bar{p}} \delta_{q}\left(\bar{p}, p^{\prime}\right)=\delta_{q}\left(p, p^{\prime}\right), \quad \bar{\delta}_{q}(p, \bar{p}) \bar{\delta}_{q}\left(\bar{p}, p^{\prime}\right)=\bar{\delta}_{q}\left(p, p^{\prime}\right), \\
\delta_{q}(p, \bar{p}) A_{q}(\bar{p})=\delta_{p, \bar{p}} A_{q}(\bar{p})=A_{q}(p), \quad A_{q}^{+}(\bar{p}) \bar{\delta}_{q}\left(\bar{p}, p^{\prime}\right)=A_{q}^{+}(\bar{p}) \delta_{\bar{p}, p^{\prime}}=A_{q}^{+}\left(p^{\prime}\right),
\end{gathered}
$$


T.K.

$$
\left(\tilde{A}_{q} \mid a_{q}^{+}\left(\bar{p}^{\prime}\right)\right) \tilde{\chi}_{q}^{-1}\left(\bar{p}^{\prime} \mid \bar{p}\right) \delta_{q}\left(\bar{p} \mid p^{\prime}\right)=0, \quad\left(\tilde{A}_{q} \mid a_{q}^{+}\left(\bar{p}^{\prime}\right)\right) \tilde{\chi}_{q}^{-1}\left(\bar{p}^{\prime} \mid \bar{p}\right) A_{q}(\bar{p})=0
$$

(см. (4.5)). Соотношения, аналогичные равенствам (4.7), будут также иметь место для функций $G_{q, E}\left(p \mid p^{\prime}\right)$ и $G_{q, E}^{-1}\left(p \mid p^{\prime}\right)$.

В соответствии с равенствами (4.2), (4.3) получаем

$$
\chi_{q}\left(p \mid p^{\prime}\right)=\left(A_{q}(p) \mid A_{q}^{+}\left(p^{\prime}\right)\right)=\delta_{q}(p, \bar{p}) \widetilde{\widetilde{\chi}}_{q}\left(\bar{p} \mid p^{\prime}\right)=\widetilde{\widetilde{\chi}}_{q}(p \mid \bar{p}) \bar{\delta}_{q}\left(\bar{p}, p^{\prime}\right),
$$

где

$$
\widetilde{\widetilde{\chi}}_{q}\left(p \mid p^{\prime}\right)=\left(\tilde{A}_{q}(p) \mid \tilde{A}_{q}^{+}\left(p^{\prime}\right)\right)=\widetilde{\chi}_{q}\left(p \mid p^{\prime}\right)-\left(a_{q}(p) \mid \tilde{A}_{q}^{\prime+}\right) \widetilde{\chi}_{q}^{\prime-1}\left(\tilde{A}_{q}^{\prime} \mid a_{q}^{+}\left(p^{\prime}\right)\right) .
$$

При условии, что матрица $\widetilde{\chi}_{q}\left(p \mid p^{\prime}\right)=\left(a_{q}(p) \mid a_{q}^{+}\left(p^{\prime}\right)\right)$ имеет обратную, т.е. $\widetilde{\chi}_{q}\left(p \mid \bar{p}^{\prime}\right) \widetilde{\chi}_{q}^{-1}\left(\bar{p}^{\prime} \mid p^{\prime}\right)=\tilde{\chi}_{q}^{-1}(p \mid \bar{p}) \widetilde{\chi}_{q}\left(\bar{p} \mid p^{\prime}\right)=\delta_{p, p^{\prime}}$, обратная матрица $\widetilde{\widetilde{\chi}}_{q}^{-1}\left(p \mid p^{\prime}\right)$ имеет вид

$$
\begin{aligned}
\tilde{\widetilde{\chi}}_{q}^{-1}\left(p \mid p^{\prime}\right)= & \tilde{\chi}_{q}^{-1}\left(p \mid p^{\prime}\right)+\tilde{\chi}_{q}^{-1}(p \mid \bar{p})\left(a_{q}(\bar{p}) \mid \tilde{A}_{q}^{\prime+}\right) \times \\
& \times\left(\tilde{\chi}_{q}^{\prime \text { int }}\right)^{-1}\left(\tilde{A}_{q}^{\prime} \mid a_{q}^{+}\left(\bar{p}^{\prime}\right)\right) \tilde{\chi}_{q}^{-1}\left(\bar{p}^{\prime} \mid p^{\prime}\right),
\end{aligned}
$$

где

$$
\begin{aligned}
\tilde{\chi}_{q}^{\text {int }} & =\tilde{\chi}_{q}^{\prime}-\left(\tilde{A}_{q}^{\prime} \mid a_{q}^{+}\left(\bar{p}^{\prime}\right)\right) \tilde{\chi}_{q}^{-1}\left(\bar{p}^{\prime} \mid \bar{p}\right)\left(a_{q}(\bar{p}) \mid \tilde{A}_{q}^{\prime+}\right)=\left(\tilde{A}_{q}^{\prime \text { int }} \mid\left(\tilde{A}_{q}^{\prime \text { int }}\right)^{+}\right), \\
\tilde{A}_{q}^{\prime \text { int }} & =\tilde{A}_{q}^{\prime}-\left(\tilde{A}_{q}^{\prime} \mid a_{q}^{+}\left(\bar{p}^{\prime}\right)\right) \tilde{\chi}_{q}^{-1}\left(\bar{p}^{\prime} \mid \bar{p}\right) a_{q}(\bar{p})= \\
& =\left(\tilde{A}_{q}^{\prime}\right)^{\text {int }}-\left(\left(\tilde{A}_{q}^{\prime}\right)^{\text {int }} \mid a_{q}\left(\bar{p}^{\prime}\right)\right) \tilde{\chi}_{q}^{-1}\left(\bar{p}^{\prime} \mid \bar{p}\right) a_{q}(\bar{p})
\end{aligned}
$$

и $\left(\tilde{A}_{q}^{\prime}\right)^{\text {int }}$ - зависящая от взаимодействия часть операторов, входяших в $\tilde{A}_{q}^{\prime}$.

Используя соотношения (4.10)-(4.12), легко убедиться, что

$$
\widetilde{\widetilde{\chi}}_{q}\left(p \mid \bar{p}^{\prime}\right) \widetilde{\widetilde{\chi}}_{q}^{-1}\left(\bar{p}^{\prime} \mid p^{\prime}\right)=\widetilde{\widetilde{\chi}}_{q}^{-1}(p \mid \bar{p}) \widetilde{\widetilde{\chi}}_{q}\left(\bar{p} \mid p^{\prime}\right)=\delta_{p, p^{\prime}}
$$

и, следовательно, обратная матрица, удовлетворяющая соотношениям (4.7), имеет вид

$$
\chi_{q}^{-1}\left(p \mid p^{\prime}\right)=\bar{\delta}_{q}(p, \bar{p}) \widetilde{\widetilde{\chi}}_{q}^{-1}\left(\bar{p} \mid p^{\prime}\right)=\widetilde{\widetilde{\chi}}_{q}^{-1}(p \mid \bar{p}) \delta_{q}\left(\bar{p}, p^{\prime}\right) .
$$

Умножая первое из соотношений (4.7) на 1 и $p_{\alpha} / m$, а второе - на 1 и $p_{\alpha}^{\prime} / m$ и суммируя их соответственно по $p$ и $p^{\prime}$, получим в согласии с равенствами (4.5) нули как в левой, так и в правой частях соотношений.

Выполнение всех необходимых условий позволяет сразу же написать уравнение для неприводимой функции $G_{q, E}\left(p \mid p^{\prime}\right)=\left(\left(a_{q}(p) \mid a_{q}^{+}\left(p^{\prime}\right)\right)\right)_{2, E}$ в стандартной форме

$$
\begin{aligned}
E G_{q, E} & \left(p \mid p^{\prime}\right)-\left\{\left\langle\left[A_{q}(p), A_{q}^{+}\left(\bar{p}^{\prime}\right)\right]_{-}\right\rangle+\left(\left(i \dot{A}_{q}(p) \mid-i \dot{A}_{q}^{+}\left(\bar{p}^{\prime}\right)\right)\right)_{3, E}\right\} \times \\
& \times \chi_{q}^{-1}\left(\bar{p}^{\prime} \mid \bar{p}\right) G_{q, E}\left(\bar{p} \mid p^{\prime}\right)=\chi_{q}\left(p \mid p^{\prime}\right) .
\end{aligned}
$$


Воспользовавшись соотношениями (4.9) и (4.2), (4.3), (4.8), из которых следует, что $G_{q, E}\left(p \mid p^{\prime}\right)=\delta_{q}(p \mid \bar{p}) G_{q, E}\left(\bar{p} \mid p^{\prime}\right)$, перепишем уравнение (4.15) в виде

$$
\begin{gathered}
\delta_{q}(p, \bar{p})\left\{E G_{q, E}\left(\bar{p} \mid p^{\prime}\right)-\left(\left\langle\left[\tilde{A}_{q}(\bar{p}), \tilde{A}_{q}^{+}\left(\bar{p}^{\prime}\right)\right]_{-}\right\rangle+\left(\left(i \dot{\tilde{A}}_{q}(\bar{p}) \mid-i \dot{\tilde{A}}_{q}^{+}\left(\bar{p}^{\prime}\right)\right)\right)_{3, E}\right) \times\right. \\
\left.\times \widetilde{\widetilde{\chi}}_{q}^{-1}\left(\bar{p}^{\prime} \mid \bar{p}\right) G_{q, E}\left(\bar{p} \mid p^{\prime}\right)-\widetilde{\widetilde{\chi}}_{q}\left(\bar{p} \mid p^{\prime}\right)\right\}=0,
\end{gathered}
$$

где мы воспользовались тем, что (см. (4.8), (4.14))

$$
\begin{gathered}
\bar{\delta}_{q}\left(p, \bar{p}^{\prime}\right) \chi_{q}^{-1}\left(\bar{p}^{\prime} \mid \bar{p}\right) G_{q, E}\left(\bar{p} \mid p^{\prime}\right)=\bar{\delta}_{q}\left(p, \bar{p}^{\prime}\right) \widetilde{\widetilde{\chi}}_{q}^{-1}\left(\bar{p}^{\prime} \mid \bar{p}\right) G_{q, E}\left(\bar{p} \mid p^{\prime}\right)= \\
=\widetilde{\widetilde{\chi}}_{q}^{-1}(p \mid \bar{p}) \delta_{q}\left(\bar{p}, \bar{p}^{\prime}\right) G_{q, E}\left(\bar{p}^{\prime} \mid p^{\prime}\right)=\widetilde{\widetilde{\chi}}_{q}^{-1}(p \mid \bar{p}) G_{q, E}\left(\bar{p} \mid p^{\prime}\right) .
\end{gathered}
$$

Поскольку $\delta_{q}(p \mid \bar{p})\left(a_{q}(p) \mid \tilde{A}_{q}^{+}\right)=0$, то уравнение (4.16) можно представить в следуюшей эквивалентной форме:

$$
\begin{aligned}
E G_{q, E}(p \mid & \left.p^{\prime}\right)-\left\{\left\langle\left[\tilde{A}_{q}(p), \tilde{A}_{q}^{+}\left(\bar{p}^{\prime}\right)\right]_{-}\right\rangle+\left(\left(i \dot{\tilde{A}}_{q}(p) \mid-i \dot{\tilde{A}}_{q}^{+}\left(\bar{p}^{\prime}\right)\right)\right)_{3, E}\right\} \times \\
\times & \widetilde{\widetilde{\chi}}_{q}^{-1}\left(\bar{p}^{\prime} \mid \bar{p}\right) G_{q, E}\left(\bar{p} \mid p^{\prime}\right)=\widetilde{\widetilde{\chi}}_{q}\left(p \mid p^{\prime}\right)+\left(a_{q}(p) \mid \tilde{A}_{q}^{+}\right) \cdot F_{q, E}\left(p^{\prime}\right),
\end{aligned}
$$

где $F_{q, E}\left(p^{\prime}\right)$ - двухкомпонентный столбец, определяемый из условий, следуюших из равенств (4.5):

$$
\left(\tilde{A}_{q} \mid a_{q}^{+}\left(\bar{p}^{\prime}\right)\right) \widetilde{\chi}_{q}^{-1}\left(\bar{p}^{\prime} \mid \bar{p}\right) G_{q, E}\left(\bar{p} \mid p^{\prime}\right)=0 .
$$

Уравнение движения для оператора $\tilde{A}_{q}(p)$ (см. (4.2)) имеет вид

$$
\begin{aligned}
i \dot{\tilde{A}}_{q}(p)= & i a_{q}(p)-\left(a_{q}(p) \mid \tilde{A}_{q}^{\prime+}\right) \widetilde{\chi}_{q}^{\prime-1} i \dot{\tilde{A}}_{q}^{\prime}=i \dot{a}_{q}(p)-\left(a_{q}(p) \mid \theta_{-q}\right)\left(\chi_{q}^{\theta}\right)^{-1} i \dot{\theta}_{q}- \\
& -\left(a_{q}(p) \mid-J_{-q}^{\prime \bar{\beta}}\right)\left(\frac{m}{q}\right)^{2} \chi_{q}^{\prime-1}(\bar{\beta} \mid \bar{\alpha}) i \dot{J}_{q}^{\prime \bar{\alpha}}- \\
& -\left(a_{q}(p) \mid-\theta_{-q}^{\prime}\right) \frac{m}{q^{2}}\left(\chi_{q}^{\theta^{\prime}}\right)^{-1} i \dot{\theta}_{q}^{\prime}
\end{aligned}
$$

В фуннкцию $\left\langle\left[\tilde{A}_{q}(p), \tilde{A}_{q}^{+}\left(p^{\prime}\right)\right]_{-}\right\rangle=\left(i \dot{\tilde{A}}_{q}(p) \mid \tilde{A}_{q}^{+}\left(p^{\prime}\right)\right)$ войдут все операторы из правой части уравнения (4.19). В “массовый оператор” $\left(\left(i \dot{\tilde{A}}_{q}(p) \mid-i \dot{\tilde{A}}_{q}^{+}\left(p^{\prime}\right)\right)\right)_{3, E}$, неприводимый относительно столбцов $A_{q}, A_{q}^{\prime}$ и $A_{q}(p)$, войдут только те части операторов, которые зависят от взаимодействия и выражаются через операторы $a_{q}(f)$ и $a_{q}(g)$.

В отличие от уравнения (2.10), гидродинамические моды из уравнения (4.17) исключены.

Уравнение для функции $G_{q, E}\left(f \mid f^{\prime}\right)($ см. (4.1)). В этом случае

$$
a(4)=a_{q}(f)=a_{p_{1}+\frac{k-q}{2}}^{+} a_{p_{2}-\frac{k}{2}}^{+} a_{p_{2}+\frac{k}{2}} a_{p_{1}-\frac{k-q}{2}}
$$


$(a(2)$ в $(2.2))$. В результате ортогонализации оператора $a_{q}(f)$ будем иметь

$$
\begin{aligned}
A_{q}(f)= & a_{q}(f)-\left(a_{q}(f) \mid A_{q}^{+}\right) \chi_{q}^{-1} A_{q}-\left(a_{q}(f) \mid A_{q}^{\prime+}\right) \chi_{q}^{\prime-1} A_{q}^{\prime}- \\
& -\left(a_{q}(f) \mid A_{q}^{+}\left(\bar{p}^{\prime}\right)\right) \chi_{q}^{-1}\left(\bar{p}^{\prime} \mid \bar{p}\right) A_{q}(\bar{p}) .
\end{aligned}
$$

Подставляя в правую часть формулы (4.20) выражение для $\chi_{q}^{-1}\left(p \mid p^{\prime}\right)(4.14)$ и используя соотношения $(4.2),(4.8),(4.11),(4.12)$, представим оператор $A_{q}(f)$ в виде

$$
A_{q}(f)=\tilde{A}_{q}(f)-\left(a_{q}(f) \mid\left(\tilde{A}_{q}^{\prime \text { int }}\right)^{+}\right)\left(\widetilde{\chi}_{q}^{\text {int }}\right)^{-1} \tilde{A}_{q}^{\prime \text { int }},
$$

где

$$
\tilde{A}_{q}(f)=a_{q}(f)-\left(a_{q}(f) \mid a_{q}^{+}\left(\bar{p}^{\prime}\right)\right) \tilde{\chi}_{q}^{-1}\left(\bar{p}^{\prime} \mid \bar{p}\right) a_{q}(\bar{p})
$$

и $\widetilde{\chi}_{q}\left(p \mid p^{\prime}\right)=\left(a_{q}(p) \mid a_{q}^{+}\left(p^{\prime}\right)\right)$. В трехкомпонентном столбце $\tilde{A}_{q}^{\prime \text { int }}\left(4.12^{\prime}\right)$ ортогональность компонент, присушая столбцу $\tilde{A}_{q}^{\prime}=\operatorname{col}\left(\theta_{q}, J_{q}^{\alpha}, \theta_{q}^{\prime}\right)$, нарушена. Ее можно легко восстановить, преобразовав $\tilde{A}_{q}^{\prime \text { int }}$ в столбец $\tilde{\tilde{A}}{ }_{q}$ с ортогональными компонентами, что сведется к замене второй компоненты $J_{q}^{\prime \alpha \text { int }}$ столбца $\tilde{A}_{q}^{\prime \text { int }}\left(4.12^{\prime}\right)$ на

$$
\tilde{\tilde{J}}_{q}^{\alpha}=J_{q}^{\prime \alpha \text { int }}-\left(J_{q}^{\prime \alpha \text { int }} \mid \theta_{q}^{\text {int }}\right)\left(\chi_{q}^{\text {int }}\right)^{-1} \theta_{q}^{\text {int }},
$$

где

$$
\theta_{q}^{\mathrm{int}}=\theta_{q}-\left(\theta_{q} \mid a_{q}^{+}\left(\bar{p}^{\prime}\right) \widetilde{\chi}_{q}^{-1}\left(\bar{p}^{\prime} \mid \bar{p}\right) a_{q}(\bar{p})\right)=h_{q}^{\mathrm{int}}-\left(h_{q}^{\mathrm{int}} \mid a_{q}\left(\bar{p}^{\prime}\right)\right) \widetilde{\chi}_{q}^{-1}\left(\bar{p}^{\prime} \mid \bar{p}\right) a_{q}(\bar{p}) .
$$

При этом вместо $\widetilde{\chi}_{q}^{\prime \text { int }}(4.12)$ получим диагональную матрицу $\widetilde{\widetilde{\chi}}_{q}=\left(\tilde{\tilde{A}}_{q} \mid \tilde{\tilde{A}}_{q}^{+}\right)$и в (4.21) будем иметь

$$
\left(\tilde{A}_{q}^{\prime \text { int }}\right)^{+}\left(\widetilde{\chi}_{q}^{\text {int }}\right)^{-1} \tilde{A}_{q}^{\prime \text { int }}=\tilde{\tilde{A}}_{q}^{+} \tilde{\widetilde{\chi}}_{q}^{-1} \tilde{\tilde{A}}_{q} .
$$

Аналогично формулам $(4.2),(4.3)$ запишем $A_{q}(f)(4.21)$ и $A_{q}^{+}\left(f^{\prime}\right)$ в виде

$$
A_{q}(f)=\delta_{q}(f, \bar{f}) \tilde{A}_{q}(\bar{f}), \quad A_{q}^{+}(f)=\tilde{A}_{q}^{+}(\bar{f}) \bar{\delta}_{q}(\bar{f}, f),
$$

где с учетом равенства (4.23) имеем

$$
\begin{aligned}
& \delta_{q}\left(f, f^{\prime}\right)=\delta_{q ; f, f^{\prime}}-\left(a_{q}(f) \mid \tilde{\tilde{A}}_{q}^{+}\right) \tilde{\widetilde{\chi}}_{q}^{-1}\left(\tilde{\tilde{A}}_{q} \mid \tilde{A}_{q}^{+}\left(\bar{f}^{\prime}\right)\right) \tilde{\chi}_{q}^{-1}\left(\bar{f}^{\prime} \mid f^{\prime}\right), \\
& \bar{\delta}_{q}\left(f, f^{\prime}\right)=\delta_{q ; f, f^{\prime}}-\widetilde{\chi}_{q}^{-1}(f \mid \bar{f})\left(\tilde{A}_{q}(\bar{f}) \mid \tilde{\tilde{A}}_{q}^{+}\right) \widetilde{\widetilde{\chi}}_{q}^{-1}\left(\tilde{\tilde{A}}_{q} \mid a_{q}^{+}\left(f^{\prime}\right)\right)
\end{aligned}
$$

И

$$
\begin{aligned}
\tilde{\chi}_{q}\left(f \mid f^{\prime}\right)= & \left(\tilde{A}_{q}(f) \mid \tilde{A}_{q}^{+}\left(f^{\prime}\right)\right)=\left(a_{q}(f) \mid a_{q}^{+}\left(f^{\prime}\right)\right)- \\
& -\left(a_{q}(f) \mid a_{q}^{+}\left(\bar{p}^{\prime}\right)\right) \tilde{\chi}_{q}^{-1}\left(\bar{p}^{\prime} \mid \bar{p}\right)\left(a_{q}(\bar{p}) \mid a_{q}^{+}\left(f^{\prime}\right)\right),
\end{aligned}
$$


$\delta_{q ; f, f^{\prime}}$ - комбинация символов Кронекера $(2.7)$ и обратная матрица $\chi_{q}^{-1}\left(f \mid f^{\prime}\right)$ определяется соотношениями

$$
\widetilde{\chi}_{q}\left(f \mid \bar{f}^{\prime}\right) \tilde{\chi}_{q}^{-1}\left(\bar{f}^{\prime} \mid f^{\prime}\right)=\widetilde{\chi}_{q}^{-1}(f \mid \bar{f}) \widetilde{\chi}_{q}\left(\bar{f} \mid f^{\prime}\right)=\delta_{q ; f, f^{\prime}}
$$

Оператор $A_{q}(f)(4.21),(4.24)$ обладает очевидным свойством (см. соотношения $(2.21),(3.13),(3.14))$

$$
\frac{1}{2 \sqrt{N} V} \sum_{f} \nu(k) A_{q}(f)=\frac{1}{\sqrt{N} V} \sum_{f} \nu_{q}^{\alpha}(f) A_{q}(f)=\frac{1}{\sqrt{N} V} \sum_{f} \nu_{q}^{h}(f) A_{q}(f)=0
$$

или $\left(\tilde{A}_{q}^{\prime \text { int }} \mid \tilde{A}_{q}^{+}\left(\bar{f}^{\prime}\right)\right) \tilde{\chi}_{q}^{-1}\left(\bar{f}^{\prime} \mid \bar{f}\right) A_{q}(\bar{f})=0$. С учетом соотношений $(4.23),(4.21)$ имеем

$$
\begin{aligned}
\left(\tilde{\tilde{A}}_{q} \mid \tilde{A}_{q}^{+}\left(\bar{f}^{\prime}\right)\right) \tilde{\chi}_{q}^{-1}\left(\bar{f}^{\prime} \mid \bar{f}\right) A_{q}(\bar{f}) & =\tilde{\tilde{A}}_{q}-\left(\tilde{\tilde{A}}_{q} \mid \tilde{A}_{q}^{\prime \text { int }+}\right)\left(\widetilde{\chi}_{q}^{\text {int }}\right)^{-1} A_{q}^{\prime \text { int }}= \\
& =\tilde{\tilde{A}}_{q}-\left(\tilde{\tilde{A}}_{q} \mid \tilde{\tilde{A}}_{q}^{+}\right) \tilde{\widetilde{\chi}}_{q}^{-1} \tilde{\tilde{A}}_{q}=0 .
\end{aligned}
$$

Последнее равенство аналогично свойству (4.5) оператора $A_{q}(p)$. Как и в случае с матрицей $\chi_{q}\left(p \mid p^{\prime}\right)(4.9),(4.14)$, находим

$$
\begin{gathered}
\chi_{q}\left(f \mid f^{\prime}\right)=\delta_{q}(f, \bar{f}) \widetilde{\chi}_{q}\left(\bar{f} \mid f^{\prime}\right)=\widetilde{\chi}_{q}(f \mid \bar{f}) \bar{\delta}_{q}\left(\bar{f}, f^{\prime}\right)= \\
=\widetilde{\chi}_{q}\left(f \mid f^{\prime}\right)-\left(\tilde{A}_{q}(f) \mid \tilde{\tilde{A}}_{q}^{+}\right) \widetilde{\widetilde{\chi}}_{q}^{-1}\left(\tilde{\tilde{A}}_{q} \mid \tilde{A}_{q}^{+}\left(f^{\prime}\right)\right), \\
\chi_{q}^{-1}\left(f \mid f^{\prime}\right)=\bar{\delta}_{q}(f, \bar{f}) \widetilde{\chi}_{q}^{-1}\left(\bar{f} \mid f^{\prime}\right)=\tilde{\chi}_{q}^{-1}(f \mid \bar{f}) \bar{\delta}_{q}\left(\bar{f}, f^{\prime}\right), \\
\chi_{q}\left(f \mid \bar{f}^{\prime}\right) \chi_{q}^{-1}\left(\bar{f}^{\prime} \mid f^{\prime}\right)=\delta_{q}\left(f, f^{\prime}\right), \quad \chi_{q}^{-1}(f \mid \bar{f}) \chi_{q}\left(\bar{f} \mid f^{\prime}\right)=\bar{\delta}_{q}\left(f, f^{\prime}\right)
\end{gathered}
$$

при условии выполнения соотношения (4.27). Действуя на обе части, например, первого из соотношений (4.31) оператором $\left(\tilde{\tilde{A}}_{q} \mid \tilde{A}_{q}^{+}\left(\bar{f}^{\prime}\right)\right) \widetilde{\chi}_{q}^{-1}\left(\bar{f}^{\prime} \mid f\right)$ (см. $\left.\left(4.28^{\prime}\right)\right)$, получаем с учетом равенств (4.28), (4.25) нуль в обеих частях равенства. В пространстве, орто-

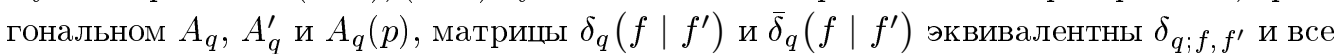
необходимые свойства (I.3.11) - (І.3.14) выполняются. Поэтому по аналогии с уравнением (4.17) можно сразу написать для функции $G_{q, E}\left(f \mid f^{\prime}\right)=\left(\left(A_{q}(f) \mid A_{q}^{+}\left(f^{\prime}\right)\right)\right)_{3, E}=$ $\left(\left(a_{q}(f) \mid a_{q}^{+}\left(f^{\prime}\right)\right)\right)_{3, E}$ уравнение

$$
\begin{aligned}
E G_{q, E}( & \left.f \mid f^{\prime}\right)-\left\{\left\langle\left[\tilde{A}_{q}(f), \tilde{A}_{q}^{+}\left(\bar{f}^{\prime}\right)\right]_{-}\right\rangle+\left(\left(i \dot{\tilde{A}}_{q}(f) \mid-i \dot{\tilde{A}}_{q}^{+}\left(\bar{f}^{\prime}\right)\right)\right)_{4, E}\right\} \times \\
& \times \widetilde{\chi}_{q}^{-1}\left(\bar{f}^{\prime} \mid \bar{f}\right) G_{q, E}\left(\bar{f} \mid f^{\prime}\right)= \\
= & \widetilde{\chi}_{q}\left(f \mid f^{\prime}\right)+\left(a_{q}(f) \mid \tilde{\tilde{A}}_{q}^{+}\right) \cdot F_{q, E}\left(f^{\prime}\right),
\end{aligned}
$$

где трехкомпонентный столбец $F_{q, E}\left(f^{\prime}\right)$ определяется из условия

$$
\left(\tilde{\tilde{A}}_{q} \mid \tilde{A}_{q}^{+}\left(\bar{f}^{\prime}\right)\right) \tilde{\chi}_{q}^{-1}\left(\bar{f}^{\prime} \mid \bar{f}\right) G_{q, E}\left(\bar{f} \mid f^{\prime}\right)=0
$$

вытекаюшего из соотношений (4.5) или из неприводимости функции $G_{q, E}\left(f \mid f^{\prime}\right)$. 
В уравнении (4.32) величины $\tilde{A}_{q}(f)(4.22)$ и $\widetilde{\chi}_{q}\left(f \mid f^{\prime}\right)$ те же, что и $A_{q}(f)(2.8)$ и $\chi_{q}\left(f \mid f^{\prime}\right)(2.9)$ во втором разделе, а “массовый оператор" имеет вид

$$
\left(\left(i \dot{\tilde{A}}_{q}(f) \mid-i \dot{\tilde{A}}_{q}^{+}\left(f^{\prime}\right)\right)\right)_{4, E}=\nu(f \mid \bar{g}) G_{q, E}\left(\bar{g} \mid \bar{g}^{\prime}\right) \bar{\nu}\left(\bar{g}^{\prime} \mid f^{\prime}\right)
$$

где

$$
G_{q, E}\left(g \mid g^{\prime}\right)=\left(\left(a_{q}(g) \mid a_{q}^{+}\left(g^{\prime}\right)\right)\right)_{4, E}=\left(\left(a_{q}(g) \mid a_{q}^{+}\left(g^{\prime}\right)\right)\right)_{\tilde{2}, E}
$$

Индекс $\tilde{2}$ в последнем из равенств (4.35) означает неприводимость функции относительно $a_{q}(p)$ и $a_{q}(f)$. Равенство (4.35) достаточно очевидно, т.к. при этом обеспечивается неприводимость относительно $A_{q}, A_{q}^{\prime}, A_{q}(p)$ и $A_{q}(f)$. Его можно получить также с помошью непосредственных вычислений. Таким образом, уравнение (4.32) отличается от $(2.11)$ только членом с $F_{q, E}\left(f^{\prime}\right)$. Уравнения для $G_{q, E}\left(g \mid g^{\prime}\right)$ и последующих функций будут те же, что и в цепочке, рассмотренной во втором разделе.

Отмечая тильдой решение уравнения $(2.11) \widetilde{G}_{q, E}\left(f \mid f^{\prime}\right)=\left(\left(a_{q}(f) \mid a_{q}^{+}\left(f^{\prime}\right)\right)\right)_{\tilde{1}, E}$, где индекс $\tilde{1}$ означает неприводимость относительно $a_{q}(p)$, запишем уравнение $(4.32)$ в виде

$$
\widetilde{\chi}_{q}\left(f \mid \bar{f}^{\prime}\right) \widetilde{G}_{q, E}^{-1}\left(\bar{f}^{\prime} \mid \bar{f}\right) G_{q, E}\left(\bar{f} \mid f^{\prime}\right)=\widetilde{\chi}_{q}\left(f \mid f^{\prime}\right)+\left(a_{q}(f) \mid \tilde{\tilde{A}}_{q}^{+}\right) \cdot F_{q, E}\left(f^{\prime}\right),
$$

из которого с учетом условия (4.33) получаем

$$
\begin{aligned}
G_{q, E}\left(f \mid f^{\prime}\right)= & \widetilde{G}_{q, E}\left(f \mid f^{\prime}\right)-\widetilde{G}_{q, E}\left(f \mid \bar{f}^{\prime}\right) \tilde{\chi}_{q}^{-1}\left(\bar{f}^{\prime} \mid \bar{f}\right)\left(a_{q}(\bar{f}) \mid \tilde{\tilde{A}}_{q}^{+}\right) \times \\
& \times \widetilde{\widetilde{G}}_{q, E}^{-1}\left(\tilde{\tilde{A}}_{q} \mid a_{q}^{+}\left(\bar{f}^{\prime}\right)\right) \widetilde{\chi}_{q}^{-1}\left(\bar{f}^{\prime} \mid \bar{f}\right) \widetilde{G}_{q, E}\left(\bar{f} \mid f^{\prime}\right)= \\
= & \widetilde{G}_{q, E}\left(f \mid f^{\prime}\right)-\left(\left(a_{q}(f) \mid \tilde{\tilde{A}}_{q}^{+}\right)\right)_{\tilde{1}, E} \widetilde{\widetilde{G}}_{q, E}^{-1}\left(\left(\tilde{\tilde{A}}_{q} \mid a_{q}^{+}\left(\bar{f}^{\prime}\right)\right)\right)_{\tilde{1}, E}
\end{aligned}
$$

где $\widetilde{\widetilde{G}}_{q, E}=\left(\left(\tilde{\tilde{A}}_{q} \mid \tilde{\tilde{A}}_{q}^{+}\right)\right)_{\tilde{1}, E}$. При $E \rightarrow \infty$ имеем

$$
\lim _{E \rightarrow \infty} E G_{q, E}\left(f \mid f^{\prime}\right)=\chi_{q}\left(f \mid f^{\prime}\right)
$$

(см. (4.29)). Функция $G_{q, E}\left(f \mid f^{\prime}\right)=\left(\left(a_{q}(f) \mid a_{q}^{+}\left(f^{\prime}\right)\right)\right)_{3, E}$ (4.37) войдет в кинетические коэффишиенты (3.24)-(3.28). Однако прежде необходимо понизить степень ее неприводимости.

\section{5. ВОССТАНОВЛЕНИЕ ПРИВОДИМЫХ ФУНКЦИЙ}

Построенная в предыдущих разделах цепочка уравнений (3.2), (3.17), (4.17), (4.32) определяет неприводимые, диагональные относительно соответствующих операторов функции Грина $G_{q, E}, G_{q, E}^{\prime}, G_{q, E}\left(p \mid p^{\prime}\right), G_{q, E}\left(f \mid f^{\prime}\right), \ldots$ Считая эти функции известными, с помощью формулы (1.7) (см. также (I.3.33)), (I.3.34)) мы можем выразить через них любую интересуюшую нас функцию с пониженной или снятой неприводимостью, диагональную или недиагональную. В качестве примера построим функции 
$\left(\left(A_{q}(p) \mid a_{q}^{+}\left(f^{\prime}\right)\right)\right)_{2, E},\left(\left(a_{q}(f) \mid a_{q}^{+}\left(f^{\prime}\right)\right)\right)_{2, E}$, через которые выражаются кинетические коэффишиенты (3.24)-(3.28), и функцию $\left(\left(a_{q}(p) \mid a_{q}^{+}\left(p^{\prime}\right)\right)\right)_{E}$, пропорциональную вариации вигнеровской функции распределения.

Полагая в (1.7) $n=3$ и $\mathcal{B}^{+}=a_{q}^{+}\left(f^{\prime}\right)$, будем иметь

$$
\begin{aligned}
& \left(\left(A_{q}(p) \mid a_{q}^{+}\left(f^{\prime}\right)\right)\right)_{2, E}= \\
& \quad=G_{q, E}\left(p \mid \bar{p}^{\prime}\right) \chi_{q}^{-1}\left(\bar{p}^{\prime} \mid \bar{p}\right)\left\{\left(A_{q}(\bar{p}) \mid a_{q}^{+}\left(f^{\prime}\right)\right)+\left(\left(i \dot{A}_{q}(\bar{p}) \mid a_{q}^{+}\left(f^{\prime}\right)\right)\right)_{3, E}\right\}= \\
& \quad=G_{q, E}\left(p \mid \bar{p}^{\prime}\right) \chi_{q}^{-1}\left(\bar{p}^{\prime} \mid \bar{p}\right)\left\{\left(A_{q}(\bar{p}) \mid a_{q}^{+}\left(f^{\prime}\right)\right)+U_{q}(\bar{p} \mid \bar{f}) G_{q, E}\left(\bar{f} \mid f^{\prime}\right)\right\},
\end{aligned}
$$

где $U_{q}(p \mid f)=\left(i \dot{A}_{q}(p) \mid A_{q}^{+}\left(\bar{f}^{\prime}\right)\right) \chi_{q}^{-1}\left(\bar{f}^{\prime} \mid f\right)$. Учитьвая определение неприводимых функций (1.5), (I.3.23), формулу (5.1) и ей сопряженную, получим

$$
\begin{aligned}
& \left(\left(a_{q}(f) \mid a_{q}^{+}\left(f^{\prime}\right)\right)\right)_{2, E}=G_{q, E}\left(f \mid f^{\prime}\right)+ \\
& \quad+\left\{\left(a_{q}(f) \mid A_{q}^{+}\left(\bar{p}^{\prime}\right)\right)+G_{q, E}\left(\bar{f} \mid \bar{f}^{\prime}\right) \bar{U}_{q}\left(\bar{f}^{\prime} \mid \bar{p}^{\prime}\right)\right\} \chi_{q}^{-1}\left(\bar{p}^{\prime} \mid \bar{p}\right) \times \\
& \quad \times G_{q, E}\left(\bar{p} \mid \bar{p}^{\prime}\right) \chi_{q}^{-1}\left(\bar{p}^{\prime} \mid \bar{p}\right)\left\{\left(A_{q}(\bar{p}) \mid a_{q}^{+}\left(f^{\prime}\right)\right)+U_{q}(\bar{p} \mid \bar{f}) G_{q, E}\left(\bar{f} \mid f^{\prime}\right)\right\} .
\end{aligned}
$$

Аналогично находим функцию распределения

$$
\begin{aligned}
& \left(\left(a_{q}(p) \mid a_{q}^{+}\left(p^{\prime}\right)\right)\right)_{E}=G_{q, E}\left(p \mid p^{\prime}\right)+\left\{\left(a_{q}(p) \mid A_{q}^{\prime+}\right)+G_{q, E}\left(p \mid \bar{p}^{\prime}\right) \bar{U}_{q, E}\left(\bar{p}^{\prime}\right)\right\} \times \\
& \quad \times \chi_{q}^{\prime-1} G_{q, E}^{\prime} \chi_{q}^{\prime-1}\left\{\left(A_{q}^{\prime} \mid a_{q}^{+}\left(p^{\prime}\right)\right)+U_{q, E}(\bar{p}) G_{q, E}\left(\bar{p} \mid p^{\prime}\right)\right\}+ \\
& \quad+\left\{\left(a_{q}(p) \mid A_{q}^{+}\right)+\left(\left(a_{q}(p) \mid A_{q}^{\prime+}\right)+G_{q, E}\left(p \mid \bar{p}^{\prime}\right) \bar{U}_{q, E}\left(\bar{p}^{\prime}\right)\right) \chi_{q}^{\prime-1} G_{q, E}^{\prime} \bar{U}_{q}\right\} \chi_{q}^{-1} G_{q, E} \times \\
& \quad \times \chi_{q}^{-1}\left\{\left(A_{q} \mid a_{q}^{+}\left(p^{\prime}\right)\right)+U_{q} G_{q, E}^{\prime} \chi_{q}^{-1}\left(\left(A_{q}^{\prime} \mid a_{q}^{+}\left(p^{\prime}\right)\right)+U_{q, E}(\bar{p}) G_{q, E}\left(\bar{p} \mid p^{\prime}\right)\right)\right\}
\end{aligned}
$$

где

$$
\begin{aligned}
U_{q, E}(p)= & U_{q}(p)+\left\{U_{q}(f)+U_{q}(\bar{g}) G_{q, E}\left(\bar{g} \mid \bar{g}^{\prime}\right) \bar{U}_{q}\left(\bar{g}^{\prime} \mid \bar{f}^{\prime}\right) \chi_{q}^{-1}\left(\bar{f}^{\prime} \mid \bar{f}\right)\right\} \times \\
& \times G_{q, E}\left(\bar{f} \mid \bar{f}^{\prime}\right) \bar{U}_{q}\left(\bar{f}^{\prime} \mid \bar{p}^{\prime}\right) \chi_{q}^{-1}\left(\bar{p}^{\prime} \mid p^{\prime}\right),
\end{aligned}
$$

здесь

$$
\begin{aligned}
U_{q} & =\left(i \dot{A}_{q} \mid A_{q}^{\prime+}\right) \chi_{q}^{\prime-1}, \\
U_{q}(n) & =\left(i \dot{A}_{q}^{\prime} \mid A^{+}\left(\bar{n}^{\prime}\right)\right) \chi_{q}^{-1}\left(\bar{n}^{\prime} \mid n\right) \quad(n=3,4,5), \\
U(n \mid n+1) & =\left(i \dot{A}(n) \mid A^{+}(\overline{n+1})\right) \chi^{-1}(\overline{n+1} \mid n+1) \quad(n=3,4) .
\end{aligned}
$$

Используя соотношения (5.3), (4.5), (4.18), легко убедиться, что

$$
\begin{aligned}
& \left(\tilde{A}_{q} \mid a_{q}^{+}\left(\bar{p}^{\prime}\right)\right) \tilde{\chi}_{q}^{-1}\left(\bar{p}^{\prime} \mid \bar{p}\right) \times \\
& \quad \times\left(\left(a_{q}(p) \mid a_{q}^{+}\left(\bar{p}^{\prime}\right)\right)\right)_{E} \tilde{\chi}_{q}^{-1}\left(\bar{p}^{\prime} \mid \bar{p}\right)\left(a_{q}(p) \mid \tilde{A}_{q}^{+}\right)=\left(\left(\tilde{A}_{q} \mid \tilde{A}_{q}^{+}\right)\right)_{E}
\end{aligned}
$$


где матрица $\left(\left(\tilde{A}_{q} \mid \tilde{A}_{q}^{+}\right)\right)_{E}$ составлена из функций Грина, построенных на операторах $\rho_{q}$ и $J_{q}^{\alpha}$, являющихся решением уравнений молекулярной гидродинамики (3.8). Снизив двукратно степень неприводимости функции (5.2), мы можем убедиться в подобной согласованности и относительно решений уравнений (3.8) для функций Грина с оператором плотности энергии $h_{q}$.

Подставляя функцию (5.2) в выражения для кинетических коэффициентов, мы придем к тому, что все интересующие нас величины будут выражены через неприводимые диагональные функции $G_{q, E}, G_{g, E}^{\prime}, G_{q, E}\left(p \mid p^{\prime}\right), G_{q, E}\left(f \mid f^{\prime}\right), \ldots$, удовлетворяющие бесконечной цепочке уравнений. Эта цепочка отличается от цепочки, рассмотренной во втором разделе, тем, что в уравнениях для функций $G_{q, E}\left(p \mid p^{\prime}\right)$ и $G_{q, E}\left(f \mid f^{\prime}\right)$ уже не содержатся уравнения гидродинамики, учтенные в (3.2), (3.17) - первых двух звеньях цепочки. Поэтому для решения уравнений, которым удовлетворяют эти функции, мы можем воспользоваться методом итераций, пренебрегая в нулевом приближении членами с коэффициентами, выражающимися через функции Грина более высокого порядка, которые к тому же по сравнению с остальными членами явно пропорциональны второй степени взаимодействия (см. (4.17), (4.32)). При этом цепочка уравнений распадается на отдельные, не связанные друг с другом уравнения. Для функции $G_{q, E}\left(f \mid f^{\prime}\right)$, через которую с учетом (5.2) выражаются кинетические коэффициенты, таким нулевым приближением будет функция, получаемая из (4.37) заменой функций $\widetilde{G}_{q, E}\left(f \mid f^{\prime}\right)$ и $\widetilde{\chi}_{q}\left(f \mid f^{\prime}\right)$ на функции $G_{q, E}^{(0)}\left(f \mid f^{\prime}\right)$ и $\chi_{q}^{(0)}\left(f \mid f^{\prime}\right)$, определяемые уравнениями $(2.22),(2.23),(2.26)-(2.28)$. Функция $G_{q, E}^{(0)}\left(f \mid f^{\prime}\right)$ дает основной вклад в кинетические коэффициенты, вычисляемые в рамках метода Чепмена-Энскога. Второй член в правой части (4.37), дающий дополнительный вклад, имеет вид дроби, числитель и знаменатель которой пропорциональны квадрату взаимодействия и не зависят от его интенсивности. Второй член в правой части равенства (5.2), возникший в результате понижения степени неприводимости, зависит также и от функции $G_{q, E}^{(0)}\left(p \mid p^{\prime}\right)$.

Детальный анализ полученных соотношений в случае конкретных потенциалов взаимодействия выходит за рамки этой статьи.

\section{6. ЗАКЛЮЧЕНИЕ}

Полученные формулы полностью решают (в рамках линейной теории) задачу построения функций распределения и линеаризованной молекулярной гидродинамики. Основная особенность метода, изложенного в трех последних разделах, состоит в том, что “главными уравнениями" являются уравнения молекулярной гидродинамики. Этим предлагаемый метод отличается от традиционного, когда за основу берется кинетическое уравнение (по сушеству, само являюшееся приближенным), а уравнения гидродинамики получаются как следствие его решения.

Метод, основанный на применении молекулярной гидродинамики, обладает рядом преимушеств по сравнению с традиционным методом.

1. Используя его, мы избавляемся от трудностей построения уравнений для моментов функции распределения, когда $h_{q}^{\text {int }} \neq 0$. При этом мы имеем уравнения для плотнос- 
тей сохраняющихся величин, пригодные не только в гидродинамической области значений $E$ и $q$, но и вне ее $($ см. $[3,4])$.

2. Согласно формуле (5.3) функция $\left(\left(a_{q}(p) \mid a_{q}^{+}\left(p^{\prime}\right)\right)\right)_{E}$ и другие подобные функции $\left(\left(\left(a_{q}(p) \mid a_{q}^{+}(f)\right)\right)_{E},\left(\left(a_{q}(f) \mid a_{q}^{+}\left(f^{\prime}\right)\right)\right)_{E}, \ldots\right)$ представляются в виде функционалов неприводимых функций, в том числе и функций Грина плотностей сохраняюшихся величин. В результате во временном представлении они явно выражаются в виде сумм членов, стремяшихся при $t \rightarrow \infty$ к нулю в соответствии с иерархией времен релаксации (кинетических, гидродинамических) и определяемых полюсами функций $G_{q, E}\left(f \mid f^{\prime}\right)$, $G_{q, E}\left(p \mid p^{\prime}\right), G_{q, E}^{\prime}, G_{q, E}$.

3. Применяемый в работе метод выходит за рамки обычной теории возмущений и является, по сушеству, методом последовательных приближений по степени учета высших корреляций.

Выбор в качестве третьего, четвертого и т.д. звеньев уравнений (4.17), (4.32) для функций $G_{q, E}\left(p \mid p^{\prime}\right), G_{q, E}\left(f \mid f^{\prime}\right), \ldots$ не является единственным. Дело в том, что если в правых частях уравнений $(3.13),(3.14)$, а также в выражениях для $i^{2} \ddot{J}_{q}^{\alpha}$ и $i^{2} \ddot{h}_{q}$ произвести суммирование по $p_{1}$ и $p_{2}$, то они выразятся через произведения операторов $\rho_{k} \rho_{-k+q}, \rho_{k} J_{-k+q}^{\alpha}, J_{k}^{\alpha} J_{-k+q}^{\beta}, \rho_{k} h_{-k+q}$, которые сами являются квазиинтегралами движения. Приняв в качестве третьего звена цепочки уравнения для функций Грина, построенных на этих операторах, мы учтем взаимодействие гидродинамических мод.

Очевидно, что изложенный в данной работе метод может быть применен и к другим физическим системам, в которых возможно выделение "медленных" и "быстрых" переменных.

\section{Список литературы}

[1] Ю. А. Церковников. ТМФ. 1999. Т. 118. № 1. С. 105.

[2] J. P. Boon, S. Yip. Molecular Hydrodynamics. N. Y.: Mc Grow-Hill Inc., 1980.

[3] Ю. А. Церковников. ТМФ. 1985. Т. 63. № 3. С. 440.

[4] Ю. А. Церковников. ТМФ. 1990. Т. 85. № 1. С. 124; № 2. С. 258.

[5] H. W. Wyld, Jr., B. D. Fried. Ann. Phys. 1963. V. 23. P. 374.

[6] C. Чепмен, Т. Каулинг. Математическая теория неоднородных газов. М.: ИЛ, 1960.

[7] I. M. de Schepper, E.G.D. Cohen, C. Buin et al. Phys. Rev. A. 1988. V. 38. P. 271.

Поступила в редакцию 11.VI.1998 г., после доработки 2.IX.1998 г. 